\title{
PVT1, a YAP1 dependent stress responsive IncRNA drives ovarian cancer metastasis and chemoresistance
}

Kevin Tabury ${ }^{1,2,3, \#, ~ M e h r i ~ M o n a v a r i a n ~}{ }^{4, \#}$, Eduardo Listik ${ }^{4}$, Abigail K. Shelton ${ }^{5}$, Alex Seok Choi ${ }^{4}$, Roel Quintens ${ }^{2}$, Rebecca Arend ${ }^{6}$, Nadine Hempel ${ }^{7}$, C. Ryan Miller ${ }^{5}$, Balázs Györrfy ${ }^{8}$, Karthikeyan Mythreye $\mathrm{e}^{3,4^{*}}$

${ }^{1}$ Department of Biomedical Engineering, University of South Carolina, Columbia, SC 29208, USA

${ }^{2}$ Radiobiology Unit, Belgian Nuclear Research Centre, SCK CEN, Mol 2400, BE

${ }^{3}$ Department of Chemistry and Biochemistry, University of South Carolina, Columbia, SC 29208, USA

${ }^{4}$ Division of Molecular Cellular Pathology, Department of Pathology, O'Neal Comprehensive Cancer Center, University of Alabama School of Medicine, Birmingham, AL, USA

${ }^{5}$ Division of Neuropathology, Department of Pathology, O'Neal Comprehensive Cancer Center, Comprehensive Neuroscience Center, University of Alabama School of Medicine, Birmingham, AL, USA.

${ }^{6}$ Department of Gynecology Oncology, University of Alabama School of Medicine, Birmingham, AL, USA.

${ }^{7}$ Department of Medicine, Division of Hematology Oncology, University of Pittsburgh School of Medicine Pittsburgh PA 15213 and Department of Pharmacology, and Obstetrics and Gynecology, College of Medicine, Pennsylvania State University, Hershey, PA, USA.

${ }^{8}$ TTK Cancer Biomarker Research Group, Institute of Enzymology, and Semmelweis University Department of Bioinformatics and 2nd Department of Pediatrics, Budapest, Hungary

\# Authors contributed equally to this work

Running title: LncRNA PVT1 drives ovarian cancer

Keywords: IncRNA PVT1, YAP1, stress response, ovarian cancer, metastasis, chemoresistance

\section{* Correspondence:}

\section{Karthikeyan Mythreye, Ph.D.}

Department of Pathology, Division of Molecular and Cellular Pathology

O'Neal Comprehensive Cancer Center

UAB, The University of Alabama at Birmingham, Birmingham, AL, USA

WTI 320B | 1824 Sixth Avenue South | Birmingham, AL 35294

E-mail: mythreye@uab.edu 


\title{
SUMMARY BLURB
}

PVT1 is a stress- responsive IncRNA regulated by YAP1, leading to increased ovarian cancer metastasis and chemoresistance, making PVT1 a promising therapeutic target.

\begin{abstract}
Metastatic growth of ovarian cancer cells into the peritoneal cavity requires adaptation to various cellular stress factors to facilitate cell survival and growth. Here we demonstrate the role of PVT1, one such stress induced long non-coding RNA, in ovarian cancer growth and metastasis. PVT1 is an amplified and overexpressed IncRNA in ovarian cancer with strong predictive value for survival and response to targeted therapeutics. We find that expression of PVT1 is regulated by ovarian tumor cells in response to cellular stress, particularly loss of cell-cell contacts and changes in matrix rigidity occurring in a YAP1 dependent manner. Induction of PVT1 promotes tumor cell survival, growth, and migration. Conversely, reducing PVT1 levels robustly abrogates metastatic behavior and tumor cell dissemination in cell lines and syngeneic transplantation models in vivo. We find that reducing PVT1 causes widespread transcriptome changes leading to alterations in cellular stress response and metabolic pathways including doxorubicin metabolism, which directly impacts chemosensitivity. Together, these findings implicate PVT1 as a promising therapeutic target to suppress metastasis and avoid chemoresistance in ovarian cancer.
\end{abstract}

\section{Introduction}

A feature of successful metastasis is the ability of cancer cells to adapt and survive under cellular stress. Mechanisms to do so include increased genomic instability, transcriptional and epigenetic changes, and acquisition of mutations [1]. Such changes are coupled with signaling pathways and gene expression alterations that facilitate cancer cell survival and thereby tumor progression. Cellular stressors impacting most cancers include changes in oxygen tension (hypoxia), changes in extracellular matrix (ECM) composition and matrix rigidity, alterations in cell-cell contacts and changes in anchorage-independent survival capabilities, oxidative, and metabolic stress as well as therapeutic treatments. Thus, defining stress dependent alterations is critical to our understanding of metastatic mechanisms.

Ovarian cancer is the fifth leading cause of cancer deaths with the highest mortality among all gynecological cancers [2]. Yet, the full etiology and pathophysiology continues to be delineated [3]. Epithelial ovarian cancer (EOC) is the most common type of ovarian cancer and is classified into different subtypes (high-grade serous carcinoma (HGS), low-grade serous carcinoma, 
mucinous carcinoma, endometroid carcinoma, and clear cell carcinoma [4] that are marked by different genome amplification and genetic instability [5]. All subtypes are exposed to cellular stressors during metastasis that involve the ovaries, the omentum, and the peritoneum. The metastatic peritoneal spread involves cell-ECM detachment, loss of cell-cell contacts, epithelialmesenchymal transition (EMT) and shedding of cells from the tumor, followed by anchorage independent survival, re-attachment to new locations and re-establishment of cell-cell contacts [6]. Primary tumors in the fallopian tube and ovaries and secondary peritoneal growths in the abdominal cavity can also be hypoxic [7], potentially further driving metastasis and chemoresistance in a feed forward manner [7, 8]. Additionally, several prior studies have reported changes to cellular stiffness during metastasis in ovarian cancer [9, 10]. Similarly, changes to the ECM and thereby matrix rigidity can also directly impact epithelial to mesenchymal transition (EMT) responses and metastasis [11].

The Human Genome Project launched the era of non-coding RNAs (ncRNAs) [12]. In these ncRNA's, small ncRNAs (<200 nucleotides) such as microRNAs, small interference RNAs and PIWI- interacting RNAs have been intensively investigated for many years [13, 14]. However, long ncRNAs (IncRNAs) (>200 nucleotides) and the contexts of their significance are still poorly understood. In ovarian cancer, several IncRNAs including MALAT1, HOTAIR and H19 are pivotal players in response to cellular stressors particularly genotoxic stress, metabolic stress and hypoxia and are also associated with tumorigenesis, metastasis and chemoresistance [15]. PVT1 is one such IncRNA located on chr8q24.21 that is expressed at low levels in normal tissues but is also designated as an oncogene due to its amplification/upregulation status in multiple cancers $[16,17]$ acting as a potential competing endogenous RNA for miRNAs [18, 19]. PVT1 has complex roles in cancers including ovarian. On one hand it has been reported to suppress cell growth [20], while in others it has been reported to promote growth [21]. Given the significant genetic instability of ovarian cancers and the ability of IncRNAs to impact metastasis through multiple mechanisms [22], a first step to precisely defining IncRNA expression outcomes is to delineate and establish contexts that regulate IncRNA expression and activity.

Here we investigate in detail the contexts and mechanism of IncRNA PVT1 expression and metastatic activities particularly in response to various stress factors in ovarian cancer. Our findings uncover PVT1 as a YAP1 dependent stress-responsive IncRNA, that can be altered transiently to drive metastasis and chemoresistance. We also discover a novel contribution of PVT1 in the regulation of doxorubicin resistance in this process. 


\section{Results}

\section{Clinical significance of PVT1 in ovarian cancer}

To evaluate in detail PVT1 expression and amplification in broad cancer types, we evaluated TCGA datasets using cBioportal [23, 24]. Analysis of somatic focal copy number gain events identified by GISTIC (v2.0) for serous ovarian cancers identified chromosome loci 8q24.21 as exhibiting the highest copy number gains (Fig 1A). Notably, MYC is located on 8q24.21 (Fig 1B). PVT1 was most significantly co-expressed with MYC, (Fig 1C and 1D - Pearson factor of 0.70 ( $P$ $=3.11$ e-46)). Among all cancers within the TCGA Firehose Legacy studies, serous ovarian cancer exhibited the highest frequency of alterations in PVT1 (42.71\%) (Fig 1E). Since serous ovarian cancers are marked by genome amplification [25], we evaluated the effect of PVT1 amplification on PVT1 expression, by comparing PVT1 amplification versus expression and found that amplification and expression were highly correlated in ovarian cancer patients (Pearson factor of $0.46 P=4.27 \mathrm{e}-17$, Fig $1 \mathrm{~F}$ ). Hence to evaluate the clinical significance of PVT1 expression changes alone, we conducted Kaplan-Meier survival analysis using KM plotter [26] in TCGA datasets that included all ovarian cancer patients with a best cutoff analysis [27]. Log-rank statistics were used to calculate the p-value and Hazard Ratio (HR). We found that patients with higher PVT1 expression had shorter overall survival (OS) (Fig 1G) and significantly shorter progression-free survival (PFS) (Fig 1H). Effects of PVT1 expression on PFS were also grade (Fig S1A) and stage dependent (Fig 1I) with an increased hazard ratio for Grade 2 (HR: 2.753, $P$ $=0.0256)($ Fig S1A) and cancer Stage $4(\mathrm{HR}=2.374, P=0.007)(F i g$ 1I). Kaplan-Meier analysis also suggests that patients with high PVT1 expression may benefit from targeted therapy (HR: $0.3344, P=0.033$ ) compared to other treatment strategies such as chemotherapy (Fig $1 \mathrm{~J}$ ). This suggests that PVT1 has predictive value for survival and may play an important role in ovarian cancer and treatment outcomes.

\section{PVT1 expression levels are altered in response to cell density changes}

Given the significant amplification of PVT1 in ovarian cancer and the apparent clinical impact of increased expression of PVT1 (Fig 1), we examined the effect of stressors, that may pertain to the metastatic trajectory of ovarian cancer on and by altering PVT1 expression in different cell lines. We first evaluated PVT1 expression at baseline using primers spanning PVT1 transcript isoforms containing - exon 1-2, exon 2-3 and exon 6-7 (Fig S1B) in a panel of human ovarian cancer (OVCA) cells lines, normal immortalized fallopian tube epithelial cells (p211) and surface epithelial cells (IOSE80) (Fig 2A). While the detectable RNA levels varied between the cell lines, SK-OV3 cells exhibited the most robust levels of detectable PVT1 across all three exons of PVT1 
(Fig 2A) consistent with prior reports of high PVT1 levels in this cell line [21, 28, 29]. Using SKOV3s, we next subjected the cells to a panel of pertinent ovarian cancer stressors including hypoxia, growth under anchorage independence and changes in cell density. We find that exposure to acute hypoxia $(0.2 \%)$ for $24 \mathrm{~h}$ led to increased PVT1 RNA levels with reproducible and significant changes across exon 6-7 (Fig 2B $P=0.0069$ ). Similarly, growing cells under anchorage independence led to fluctuations in levels of PVT1 as compared to growth under attached conditions, but this comparison did not reach statistical significant (Fig 2C). In contrast to the modest changes observed in response to the above-described stressors, we found reproducible and significant changes in PVT1 RNA levels upon changes in cell density (Fig 2D). Specifically, switching cells between high and low-density growth revealed marked and significant differences in PVT1 RNA levels, with increases across all exons seen in low density conditions (Fig 2D $(P<0.0001)$ ). Cell-cell contact, or absence thereof (low density) was confirmed by phase contrast microscopy (Fig 2D, inset images). The effect of changing cell density on PVT1 levels was recapitulated in other cell lines as well, including human high grade serous OV90 across one exon (Fig S1C) and across exons 2-3 in the mouse ID8 cell line both in the presence and absence of Trp53 (ID8 Trp53-/-), the most commonly altered gene in OVCA (Fig. 2E and 2F) $(P=0.0112$ and 0.0044 respectively).

However, in a subset of ovarian cancer cell lines including OVCAR3 and OVCAR420, cell density alterations failed to induce changes in PVT1 expression (data not shown). We speculated that several serous ovarian cancer cell lines grow in clusters in the presence of cell-cell contacts, unlike SK-OV3 cells which exhibit mesenchymal morphology and growth patterns [30]. To test if PVT1 changes in response to cell density were a feature of mesenchymal cells, we induced EMT in EOC OVCAR3 and OVCAR420 epithelial cells with TGF $\beta$-1. EMT was confirmed by increases in ZEB1 and SNAIL1 expression (Fig S1D). Post EMT mesenchymal cells indicated as OVCAR3$M$ and OVCAR420-M, were then subjected to either high density or low-density growth conditions followed by evaluation of PVT1 levels. We find that PVT1 levels across all exons were increased under low density in both OVCAR3-M and OVCAR420-M cells (Fig 2G-H) (OVCAR3: exon 1-2 $P$ = 0.002; exon 2-3 $P=0.0013$; exon 6-7 $P=0.0134$; OVCAR420: exon 2-3 $P=0.0229$; exon 6-7 $P=0.0086$ ), mimicking the cell density response in SK-OV3 and ID8 cells (Fig 2D-F). These data indicate that isogenic mesenchymal cells are particularly sensitive to cell density associated PVT1 changes as compared to their epithelial counterparts. To evaluate if PVT1 levels correlate with a mesenchymal gene signature in ovarian cancer patients we examined a panel of EMT genes and PVT1 in the ovarian cancer TCGA data. We find a positive correlation between PVT1 and EMT 
associated genes (Fig 2I) suggesting a strong correlation between post EMT regulation of PVT1 in ovarian cancer.

\section{PVT1 is regulated by YAP1 in ovarian cancer}

Cell density changes can lead to alterations in the cell cycle and concomitant changes to the Hippo pathway, a key modulator of cell survival in response to cellular stressors [1-3] and a key negative regulator of YAP1 in various models [31, 32]. Based on the cell density associated changes in PVT1 levels, we first evaluated if SK-OV3 cells under high density retain active Hippo signaling, as measured by nuclear YAP1 localization [31, 32, 33]. We found nuclear accumulation of YAP1 in cells plated under low density, with significant exclusion under high density (Fig 3A and 3B $P<0.0001$ ). Additionally, YAP1 target genes including CTGF and CYR61 were significantly upregulated (CYR61 $P<0.0001$; CTGF $P=0.0084$ ) under low density compared to high density growth indicative of YAP1 function and activity under low density (Fig 3C).

To mechanistically extend the correlative observations of PVT1 RNA changes with YAP1 localization under low density, we tested if changing matrix rigidity would impact PVT1 RNA levels independent of the cell density associated changes. We first utilized fibronectin conjugated polyacrylamide hydrogels with an elastic modulus of $0.5 \mathrm{kPa}$ (soft), $8 \mathrm{kPa}$ (stiff) and normal plastic plates (in the order of $\mathrm{GPa}$ ). We find that increasing matrix rigidity leads to increased PVT1 RNA levels (Fig 3D, $0.5 \mathrm{kPa}$ vs $8 \mathrm{kPa}$ : exon 1-2 $P=0.2523$; exon 2-3 $P=0.6295$; exon 6-7 $P=0.4684$; 0.5 kPa vs 8 GPa: exon 1-2 $P<0.0001$; exon 2-3 $P<0.0001$; exon 6-7 $P<0.0001$; 8 kPa vs GPa: exon 1-2 $P=0,0002$; exon 2-3 $P<0.0001$; exon 6-7 $P=0.0002$ ). To confirm the direct correlation between YAP1 activity and substrate rigidity, CYR61 and CTGF mRNA expression was monitored and was found to be upregulated with increasing matrix rigidity (Fig 3E). Serum is a known inhibitor of the Hippo pathway [34] and leads to increased YAP1 activity. To test if depleting serum from low density cells when Hippo signaling is low and PVT1 is elevated, would lower PVT1 expression, we serum starved low density population of cells and evaluated PVT1 levels. We find that serum starved low density cells, further reduced their levels of PVT1 (Fig 3F) and at the same time also reduced YAP1 activity as indicated by the decrease of CYR61 and CTGF mRNA expression (Fig. 3G). These data point to regulation of PVT1 co-incidentally with, or dependent on YAP1 activity.

To directly test if PVT1 expression depended on YAP1 levels, we used shRNAs' to lower YAP1 levels $(\mathrm{Fig} 3 \mathrm{H})$ or inhibited the YAP1-TEAD association using the small molecule inhibitor verteporfin (Fig 3I) [35]. We find that specific reduction of YAP1 or the use of verteporfin significantly reduced PVT1 levels compared to control cells (Fig $3 \mathrm{H}, 3 \mathrm{I}$ ). Based on this apparent 
dependency of PVT1 expression on YAP1 function, we next evaluated the clinical relevance of the PVT1-YAP1 relationship. We found a strong correlation between an inactive Hippo gene signature (where YAP1 target gene signatures are expressed), and PVT1 expression in TCGA ovarian cancer datasets (Fig $3 \mathrm{~J}$ ). Together, these observations indicate that YAP1 is a regulator of PVT1 with functional correlations in ovarian cancer patients.

\section{PVT1 confers survival and pro-metastatic advantages to OVCA cells in vitro and in vivo}

To test the effects of altering PVT1 levels on tumor cell behavior in vitro, we first used shRNAs (Fig 4A-B) to reduce PVT1 levels in both human SK-OV3 cells and mouse ID8 Trp53 -/- cells. We found that lowering PVT1 resulted in reduced proliferation (MTT assay $-P<0.0001$ ), migration potential of the tumor cells (transwell migration assay $-P=0.003$ ) and colony formation and clonogenic survival $(P=0.0088)$ (Fig 4C-E). Similar results were obtained upon reducing PVT1 levels in mouse ID8 Trp53 -/- cells with shRNA, leading to significant reduction of proliferation (MTT assay $-P<0.0001$ ), as well as migration potential (transwell migration assay $-P=0.0163$ ) (Fig 4F-G). Conversely, to evaluate the effect of increasing PVT1 expression, human PVT1 was cloned into pcDNA3.1(+) vector and expressed in SK-OV3 cells. Exogenous PVT1 expression was evaluated by RT-qPCR and fluorescence in situ hybridization (FISH, Fig 4H, 4I). We found that overexpressing PVT1 resulted in increased proliferation (Fig $4 \mathrm{~J}-P<0.0001$ ), increased colony formation in a long term clonogenicity assay $(P<0.0001)$, and increased migration (transwell migration assay - $P=0.0007$ ) (Fig 4J). These data suggest that while mouse and human PVT1 sequences vary between species [36], both have likely conserved functions [37] and promote tumorigenic behavior of cell lines.

Peritoneal growth and metastasis involves changes in cell-cell and cell-ECM contacts and survival of single cells that can attach to peritoneal organs [6] [38]. We therefore tested if reducing PVT1 levels altered intraperitoneal tumor growth. We reduced Pvt1 expression in ID8 Trp53-/cells with shRNAs targeting Pvt1 (shPvt1) or non-targeting control (shctrl) (Fig 5A.i) and injected $5 \times 10^{6}$ viable cells within $96 \mathrm{~h}$ of Pvt1 knockdown into the peritoneal cavity of C57BL/6J mice ( $\mathrm{n}=$ 12). Half the mice $(n=6)$ were sacrificed after 4 weeks to capture earlier effects of Pvt1 expression differences (mid-point of tumor growth as determined from pilot studies) and the remaining 6 mice were sacrificed at 8 weeks when they were moribund (Fig 5B). At the 4 week time point, we found that mice receiving control cells had already developed small omental and intraperitoneal lesions with observable tumor growth (Fig 5A. ii,ii) compared to mice receiving shPvt1 cells (Fig. 5A, black arrows). Omental weights were also measurably higher in mice receiving shctrl cells (Fig 5A.iv). The reduction in peritoneal lesions persisted over time, as shPvt1 cells had visibly (Fig 
5B.i,ii) and measurably reduced overall tumor burden at 8 weeks (Fig 5B.iii, n=6 for each group with one shctrl receiving mouse moribund prior to 8 weeks). Assessment of the omentum, ovary, and peritoneal wall, all primary target tissues of metastatic ovarian cancer [39], revealed that cells expressing Pvt1 (shctrl) had seeded the peritoneum efficiently and invaded into omental tissue (Fig 5B.i,ii). In contrast, lowering Pvt1 in shPvt1 cells led to significantly lower amounts of ascites fluid (Fig 5B.iv $P<0.0001$ ) and abdominal girth (Fig 5B.v $P<0.0001$ ). These striking differences indicate that reducing Pvt1 leads to significantly less intraperitoneal tumor burden and demonstrates the critical role of PVT1 in ovarian cancer growth in vivo.

\section{PVT1 impacts global gene expression and regulates sensitivity to doxorubicin.}

Since PVT1 is a IncRNA with likely broad range effects, we evaluated the impact of PVT1 on global gene expression by performing RNA sequencing in human SK-OV3 cells upon silencing PVT1 using siRNAs (siPVT1). We found that 450 protein coding genes were differentially expressed between control (siCon) and siPVT1 cells with 50 additional genes found to be nonprotein coding. The top 50 differentially expressed genes include both downregulated and upregulated genes in response to reducing PVT1 by siRNAs (Fig 6A). Lowered PVT1 levels were confirmed in the RNA-seq dataset (Fig S2A) as well as by RT-qPCR in biological replicates (Fig S2B). Furthermore, principal component analysis (PCA) confirmed PVT1 siRNA status as a factor influencing the gene expression profiles (Fig S2C). The gProfiler web analysis tool was used to investigate all significant genes $(n=450)$. We find that PVT1 siRNA leads to substantial changes in the biological pathways associated with several stress responses and metabolism, specifically genes associated with doxorubicin metabolism (Fig 6B, C). Gene set enrichment analysis confirmed the "doxorubicin metabolism process" (Fig 6D) with genes such as AKR1C1, AKR1C2 and AKR1B10 being significantly downregulated in the siPVT1 samples (Fig 6C). Expression changes in these genes were also validated and confirmed by RT-qPCR (Fig 6E). We hence tested if siRNA to PVT1 sensitized SK-OV3 cells to doxorubicin. We found a 1.5-fold reduction in the $I_{50}$ to doxorubicin in siPVT1 as compared to siControl SK-OV3 cells (Fig 6F). Since human SK-OV3 cells do not express p53 [39], we evaluated if siPVT1 sensitized cells to doxorubicin even in the presence of p53 as the cell density dependent regulation of Pvt1 was largely p53 independent (Fig2E,F). To test this, we used mouse ID8-IP2 cells that express wild type Trp53 [40] and evaluated doxorubicin sensitivity. We found that siRNA to Pvt1 significantly lowered doxorubicin $\mathrm{IC}_{50}$ in these cells as well (Fig 6F) indicating conserved functions across species and likely independent of the p53 status. These findings demonstrate a novel correlation between PVT1 and doxorubicin resistance in ovarian cancer. 


\section{Discussion}

Here we demonstrate that PVT1 is a contextual oncogenic IncRNA, amplified along with MYC, and a prognostic indicator in ovarian cancers that is dynamically altered in expression primarily in response to cellular stressors. Notably cell density and matrix stiffness changes, both of which converge on pathways associated with YAP1, lead to regulation of PVT1. Our study is the the first to report such a mechanism and suggest a potential feedforward relationship between YAP1 and PVT1.

We find a strong correlation between PVT1 and MYC in ovarian cancer, as has been previously documented [17, 41, 42]. Genome-wide association studies have identified chromosome 8q24.21 as a cancer risk locus in multiple cancers [43], but also specifically in ovarian cancer [44, 45]. Besides harboring MYC, dysregulation and amplification of which is found in many human cancers [46], the 8q24.21 locus also harbors IncRNA PVT1 $53 \mathrm{~kb}$ downstream of MYC. Bioinformatic analysis of TCGA datasets confirmed that chromosome 8q24.21 exhibits the highest copy number amplifications and that copy number alteration (CNA) frequency of PVT1 is among the highest in ovarian cancer (Fig 1), highlighting the significance and relevance of PVT1 to this cancer. Further, PVT1 expression is also correlated with patient survival outcomes. Ovarian cancers are mostly detected at an advanced stage which is one of the causes of poor survival. Our findings are consistent with other reports of higher PVT1 mRNA expression in stage 3 compared to other stages [47, 48]. Interestingly, the impact of PVT1 expression differs for earlystage patients (stage II) where low PVT1 expression is correlated with poor progression free survival (Fig 1I). As ovarian cancers are marked by genome amplification, we investigated the correlation between PVT1 amplification and expression. Our data indicated a strong positive correlation between PVT1 amplification on chr 8q24.21 and expression in the TCGA cohort of primary patient tumors (Fig 1F).

We demonstrate here for the first time that PVT1 RNA expression is modulated by cell density, hypoxia, and matrix rigidity in both human and mouse ovarian cancer cell lines. Increases in PVT1 RNA expression under hypoxia have been previously described in non-ovarian cancers [49-51], but were somewhat modest in the ovarian cancer cell lines we tested here (Fig 2B). The most consistent and reproducible stressors that alter PVT1 expression are changes to cell density and mesenchymal status across cell lines, regardless of their baseline PVT1 levels (Fig 2A). The Hippo signaling pathway is altered in ovarian cancer and causes increased nuclear YAP1 and tumorigenesis [52, 53]. Interestingly, modulation of PVT1 RNA expression after exposure to the 
stressors followed the RNA expression of YAP1 canonical target genes CTGF and CYR61 [33, 52], indicating a correlation between YAP1 nuclear localization and PVT1 RNA expression, that we also observed in patient data (Fig 3J). PVT1 expression levels were directly dependent on YAP1 levels as investigated through YAP1 knockdown as well as through disrupting YAP/TEAD interaction $[33,54]$. In the context of EMT, tumors often exhibit a spectrum of EMT states. Indeed, cell lines such as OVCAR3 and OVCAR420 exhibit a more epithelial phenotype, whereas SKOV3s exhibit a more intermediate to mesenchymal phenotype [55] and were found to be responsive to cell density dependent changes in PVT1. YAP1 plays a dual role as both inducer and effector of EMT [56] and has been shown to translocate from the cytoplasm to the nucleus after EMT induction through TGF $\beta$ treatment [57]. Consistently, we observed a significant sensitivity of EMT induced cells to cell density dependent induction of PVT1 as seen in post EMT OVCAR3 and OVCAR420 cells that were rendered sensitive to cell density dependent PVT1 changes (Fig 2). While it is likely that the PVT1 promoter possesses a TEAD binding site [58] the complex of YAP-TEAD on PVT1's promoter has not yet been reported and may involve indirect regulatory mechanisms as well. Little is known about modulators of PVT1 expression itself. Prior studies have demonstrated MYC [17], FOXM1 [59], STAT3 [60] and TP53 [61] as regulators that can bind to PVT1's promoter. For instance, it has been shown that FOXM1, which binds to PVT1's promoter to induce PVT1 RNA expression [59], is a downstream effector of YAP1 [62]. Thus whether regulation is occurring here, directly or indirectly at the promoter remains to be determined. A surprising observation is the lack of a role of p53 in cell density dependent changes in Pvt1. Several prior studies have demonstrated PVT1 as a direct p53 target $[63,61]$. Our findings suggest that the YAP1 dependent mechanism of PVT1 regulation may be p53 independent.

Functional assays with gain or loss of PVT1 RNA expression demonstrated PVT1's role in promoting survival and chemoresistance in ovarian cancer cell lines and during intra peritoneal tumor growth, highlighting a pro- metastatic role for Pvt1 in ovarian cancer. Strikingly short-term knockdown of Pvt1 (within 96 hours of shRNA administration) was sufficient to cause a significant reduction in tumor burden, suggesting that transient dynamic changes occurring during disease progression may be adequate at impacting disease outcomes.

In the context of the models used here, transcriptomic analysis revealed a novel contribution of PVT1 in the regulation of aldo-keto reductases including AKR1C, AKR1C2, and AKR1B10. Aldo-keto reductases (AKR) have been demonstrated to regulate the metabolic processing of chemotherapeutic drugs such as doxorubicin, cisplatin, and paclitaxel, among others, and to cause chemoresistance in multiple cancers $[64,65]$. AKR1B10 has been linked to cellular resistance to doxorubicin by inducing the conversion of doxorubicin to doxorubicinol which 
is significantly less toxic [66]. AKR1B10 has also been reported to have an oncogenic role across different cancer types [67]. Indeed, lowering Pvt1 increased the sensitivity to doxorubicin. Here again, the effect of Pvt1 on the sensitivity to doxorubicin was Trp53 independent. Therefore, targeting PVT1 to suppress the level of these AKR enzymes and consequently reducing inhibitory concentration of doxorubicin could pave the way toward minimizing the dose dependent cardiotoxicity of doxorubicin [68]. The exact mechanism by which PVT1 regulates AKRs expression remains to be elucidated.

In summary the broad impact of PVT1 on multiple pathways suggest that blocking PVT1 may be an attractive target to simultaneously suppress multiple pathways. Few tools to silence IncRNAs are currently available (RNA interference, antisense oligonucleotides and genome editing (CRISPR/Cas9 system) but none have been clinically employed for PVT1 [69] and warrant further investigation for ovarian cancers.

\section{Materials and Methods}

\section{Cell lines and reagents}

Human ovarian cancer cell lines SK-OV3 and OV-90-CRL-11732 ${ }^{\mathrm{TM}}$ were purchased from ATCC. OVCAR3 and OVCAR5 were obtained from NIH (NCl-60). OVCAR420 and HeyA8 cells were a gift from Susan K Murphy. P211 were derived as described previously [70], [71], IOSE80 cells were received from the Canadian tissue bank. Mouse ovarian surface epithelial cell line ID8 and ID8-IP2's were a kind gift from Jill K. Slack-Davis and ID8 Trp53-/- were a kind gift from lain McNeish. SK-OV3, OVCAR3, OVCAR5, OVCAR433, OVCAR420, and HeyA8 were cultured in RPMI-1640 (corning 10-040-CV) supplemented with 10\% FBS (Corning 35-010-CV) and 100 units $/ \mathrm{mL}$ penicillin, $100 \mu \mathrm{g} / \mathrm{mL}$ streptomycin at $37^{\circ} \mathrm{C}$ in a humidified incubator containing $5 \% \mathrm{CO} 2$. ID8 and ID8 Trp53 -/- were cultured in DMEM (Corning 10-017-CV) supplemented with 4\% FBS, $5 \mu \mathrm{g} / \mathrm{mL}$ insulin, $5 \mu \mathrm{g} / \mathrm{mL}$ transferrin, $5 \mathrm{ng} / \mathrm{mL}$ sodium selenite, and 100 units $/ \mathrm{mL}$ penicillin, 100 $\mu \mathrm{g} / \mathrm{mL}$ streptomycin at $37^{\circ} \mathrm{C}$ in a humidified incubator containing 5\% CO2. P211 and IOSE80 were cultured in DMEM supplemented with $10 \%$ FBS plus 100 units $/ \mathrm{mL}$ penicillin, $100 \mu \mathrm{g} / \mathrm{mL}$ streptomycin. OV90 cells were cultured in a 1:1 mixture of MCDB 105 medium (Sigma Aldrich M6395-1L) and Medium 199 (Sigma Aldrich M5017-10X1L) containing 15\% fetal bovine serum plus 100 units $/ \mathrm{mL}$ penicillin and $100 \mu \mathrm{g} / \mathrm{mL}$ streptomycin. All cell lines were grown at $37^{\circ} \mathrm{C}$ in a humidified incubator containing $5 \% \mathrm{CO}_{2}$ and

Antibodies: monoclonal mouse anti-YAP1 (Santa Cruz Biotechnology, sc-101199), Alexa Fluor 488 goat anti-mouse IgG, H+L (Invitrogen, A11001). 
Reagents: PolyHema (Sigma, P3932) was used to generate low attachment plates for the anchorage-independent assay; fibronectin (Recombinant Human Fibronectin R\&D Systems, 8258-FN-050); bovine serum albumin (BSA, BioExpress, 0903-5G); crystal violet (VWR, AA22866-14); PolyBrene (Santacruz Biotechnology, sc-134220); DMSO (Fisher Scientific, BP231-100); Paraformaldehyde (Avantor, S898-07); Triton-X100 (AMRESCO, 0694-1L); absolute ethanol (Decon Laboratories); epithelial to mesenchymal transition (EMT) was induced with 200 pM of Human recombinant TGF-beta 1 (R\&D Systems, 240-B); verteporfin (Sigma Aldrich, SML0534-5MG); Paclitaxel (Fisher Scientific, 328420010); Doxorubicin (Fisher Bioreagent, BP25176-10); Cisplatin (Medchem Express, HY-17394).

\section{Bioinformatic analysis}

Somatic focal copy number gain events identified by Genomic Identification of Significant Targets in Cancer (GISTIC) for TCGA ovarian serous cystadenocarcinoma were recovered from firebrowse (http://firebrowse.org/). Genome data viewer was used to investigate genes located in chromosome 8q24.21 (https://www.ncbi.nlm.nih.gov/genome/gdv/). TCGA data for ovarian serous cystadenocarcinoma (TCGA, Firehose Legacy, RNA Seq V2, 307 samples) was recovered from cBioportal (https://www.cbioportal.org/) and used to investigate mRNA expression, mRNA co-expression and copy number alterations (CNA). Survival data was generated from KM Plotter using the TCGA datasets, all patients and the best cutoff setting [26, 27]. Log-rank statistics were used to calculate the $p$-value $(P)$ and Hazard ratio $(H R)$.

Z-score normalized RNA-seq data from ovarian cancer patients were retrieved from TCGA datasets. Elements from the "GO Hippo signaling" and "GO Epithelial to Mesenchymal transition" genesets [72] were filtered, and Hippo signaling score or EMT score was obtained from the direct sum of the gene's z-scores. Spearman rank correlation test was performed to assess whether PVT1 expression correlates to each obtained score. PVT1-high and -low groups of patients were also checked for mRNA expression of each gene within each geneset. Each group contained patients with the highest or lowest $10^{\text {th }}$ percentile of PVT1 expression.

\section{Generation of stable overexpression/knockdown cells}

For PVT1 and YAP1 knockdown, SK-OV3 and ID8 Trp53 -/- cells were infected with $10 \mathrm{MOI}$ of shRNAs or controls. All virus production was performed by the Functional Genomics Core (FGC) of the Center for Targeted Therapeutics, University of South Carolina. In short, HEK293-FT cells, cultured in DMEM (high glucose) media with 10\% FBS, 2 mM L-glutamine, penicillin (100 IU/ml), and streptomycin $(100 \mathrm{mg} / \mathrm{ml})$ were transiently transfected with specific lentiviral constructs and 
the packaging/envelope plasmids pMD2.G and psPAX2. The Virus-containing medium was collected at $48 \mathrm{~h}$ and $72 \mathrm{~h}$ after transfection and centrifuged at $200 \mathrm{~g}$ for $5 \mathrm{~min}$ at $4{ }^{\circ} \mathrm{C}$. The medium was filtered with $0.45-\mu \mathrm{m}$ PES Syringe Filter and centrifuged at $21,000 \mathrm{~g}$ for $16 \mathrm{~h}$ at $4{ }^{\circ} \mathrm{C}$. Pellets of lentivirus were re-dissolved in PBS at one-hundredth of the original volume. Lentiviruses were then diluted with fresh culture media to transduce target cells in the presence of $10 \mu \mathrm{g} / \mathrm{ml}$ PolyBrene (hexadimethrine bromide). Sequences for the corresponding shRNAs are in Table 1. For the overexpression construct, the complete linear RNA PVT1 sequence (1969 bp) was inserted in a pcDNA3.1(+) vector (SC1691 - GenScript). Control samples were achieved using an empty pcDNA3.1(+) plasmid. For YAP1, two shRNAs that include multiple transcript variants were inserted in a pLKO.1 vector (TRCN0000107265 and TRCN0000107268). Control samples were achieved using a TRCN scr PLKO.1 plasmid. The efficiency of knockdown or overexpression was performed by RT-qPCR (list of primers are in Table 2).

For transient PVT1 knockdown using siRNA, SK-OV3 cells were cultured to $50 \%$ confluence in 6 well plates. Human siRNA for PVT1 was achieved using a mixture of 4 siRNA (ACCUAUGAGCUUUGAAUAA; GAGAACUGUCCUUACGUGA; CUUCAACCCAUUACGAUU and GUACGAACUUCAUCGCCCA) (smartpool R-029357-00-0005 - Dharmacon). Control samples were achieved using Lincode Non-targeting Pool (D-001320-10-05 - Dharmacon). 50 nM Pooled siRNA to human PVT1 or non-targeting siRNA from Dharmacon ） were used to transfect SK-OV3 cells for 48 hours in full serum media carefully maintaining cell confluence to not exceed approximately 80\%. Lipofectamine RNAiMAX Transfection Reagent (ThermoFisher 13778075) was used to facilitate the transfection. This was followed by RNA extraction and verification of knockdown using primers to PVT1.

\section{Functional assays}

For transwell migration assay, $8 \mu \mathrm{m}$-pore membranes (Greiner bio-one, 662638) were coated with $10 \mu \mathrm{g} / \mathrm{ml}$ fibronectin. A total of 20,000 cells were suspended in $100 \mu \mathrm{l}$ serum-free medium and added to the upper chamber of each transwell. The lower chamber was filled with $600 \mu$ complete medium and incubated in a $\mathrm{CO}_{2}$ incubator at $37^{\circ} \mathrm{C}$ for $6 \mathrm{~h}$, after which fixation and staining occurred. For clonogenicity assay, 200 cells were cultured in each well of a 6-well plate in complete medium for 10 days, and after cells were fixed and stained. Imaging was done using EVOS M7000 inverted microscope (Thermo Fisher). Quantitation was performed by manual counting. For MTT assay, 1,000 cells were seeded in a 96-well plate for $24 \mathrm{~h}$, then a final concentration of $1 \mathrm{mM}$ MTT was added. The plates were incubated in a $37^{\circ} \mathrm{C}$ incubator for 2.5 
hrs, and DMSO was added to dissolve the formazan crystals. Absorbance was measured using a Synergy HT plate reader at $570 \mathrm{~nm}$.

\section{Cell density assay}

Low cell density was achieved in seeding 35,000 cells per well in a 6-well plate. High density was achieved in seeding 210,000 cells per well in a 24 -well plate.

\section{Hypoxia assay}

A hypoxia chamber was used to regulate the oxygen gas levels in the incubator to $0.2 \%$. A total of 20,000 cells were seeded in 24 -well plates for $24 \mathrm{~h}$ under hypoxia or normoxia.

\section{Matrix stiffness assay}

Easy Coat ${ }^{\mathrm{TM}}$ hydrogels Softwell ${ }^{\circledR}$ plates with $0.5 \mathrm{kPa}$ and $8 \mathrm{kPa}$ were obtained from Matrigen (SW6-EC-0.5 EA, SW6-EC-8 EA) and regular 6-well plates were coated with $10 \mu \mathrm{g} / \mathrm{mL}$ fibronectin prior to use. Cells were seeded at a density of 100,000 in a 6-well plate and incubated for $24 \mathrm{~h}$.

\section{IC50 determination.}

SK-OV3 and ID8-IP2 cells were cultured in 96-well plates at a density of 2,500 cells per well for $24 \mathrm{~h}$. Then the medium was replaced with fresh medium containing Doxorubicin. After $72 \mathrm{~h}$ incubation with drugs, Sulforhodamine B (SRB, Alfa Aesar, A14769-14) assay was performed as previously described [73]. IC50 was calculated using IC50 calculator (ATT Bioquest Inc 2021, https://www.aatbio.com/tools/ic50-calculator)

\section{RNA Fluorescence In Situ Hybridization and Immunofluorescence}

PVT1 visualization and localization were performed using a ViewRNA cell plus assay probe for PVT1 (ThermoFisher, VA4-3082274-VCP). The probe set per manufacturer covers region 4231392 and is designed to hybridize with human PVT1 specifically). Cells were seeded onto a NUNC 8-well chamber slide. DapB was used as a negative control, whereas GAPDH was used as a positive control. The procedure was performed as described by the manufacturer with no modifications. Images were acquired with a ZEISS LSM 800 confocal.

For standard immunofluorescence, cells were fixed in 4\% paraformaldehyde for 15 minutes and permeabilized with $0.3 \%$ Triton $X$ for 10 minutes at room temperature. Blocking was performed with $5 \% \mathrm{BSA}$, and cells were then incubated with a 1:100 dilution of anti-YAP1 overnight at $4{ }^{\circ} \mathrm{C}$. Then, the primary antibody was conjugated to Alexa 488, at $1: 200$, for $1 \mathrm{~h}$ at room temperature in the dark. Images were obtained using an EVOS M7000 microscope. Image $\mathrm{J}$ was used to perform the quantification. 


\section{Animal studies}

All mouse studies were performed in accordance with the Institutional Animal Care and Use Committee at the University of Alabama Birmingham. Female C57BL/6J mice were obtained from the Jackson laboratory. Five million ID8-Trp53 -/- shCtrl and shPvt1 were injected intraperitoneally in mice ( $n=12$ per group). Mice were monitored daily with girth and weight measurement taken weekly. Animals were sacrificed after either 4 weeks $(n=6)$ or 8 weeks $(n=6)$. At necropsy, ascites, if present, were collected and volumes measured, tumor weights in the omentum and other organs were recorded and collected when possible. For microscopic analysis of tissues, formalinfixed tissues were processed, paraffin-embedded, and sectioned at $5 \mu \mathrm{m}$ thickness and H\&E stained at UAB's histology core.

\section{RNA-sequencing}

Library preparation was performed on purified, extracted RNA using a KAPA mRNA HyperPrep Kit (Kapa, Biosystems, Wilmington, MA) according to the manufacturer's protocol. High throughput sequencing with 75-bp single-end reads was performed on an Illumina NextSeq 550 using an Illumina NextSeq 500/550 High Output Kit. Reads were aligned to the human transcriptome GENCODE v35 (GRCh38.p13) using STAR and counted using Salmon [74, 75]. Normalization and differential expression analysis were performed using the $\mathrm{R}$ package DESeq2 v1.34 [76]. Genes where there were fewer than three samples with normalized counts less than or equal to five were filtered out of the final data set. Benjamini-Hochberg-adjusted $p$ value of $p<0.05$ and log 2 fold change of 1 were the thresholds used to identify differentially expressed genes between treatment conditions. Pathway enrichment analysis was performed using GSEA [77, 78].

\section{Statistical analysis}

Xenograft data were analyzed using parametric statistics. All real time PCR's are relative quantitative RT-PCR's (hereby referred to as RT-qPCR) and are a combined quantitation of a independent biological trials (indicated in legends) assayed in triplicate. All statistical analyses were conducted with GraphPad Prism Software and utilized tests referred to in the figure legends

\section{Data availability}

The authors confirm that all data supporting the findings of this study are available within the paper and supplementary data. RNA-seq data have been deposited in the NCBI-Gene Expression Omnibus (GEO) database under the accession ID GSE185933. 


\section{Author contributions}

K.T., M.M. and A.C. performed experiments. K.T., M.M., E.L., N.H., B.G., R.Q. analyzed data.

K.T., M.M. and K.M. designed the study, analyzed the data, and supported the work. K.T.; M.M. and K.M. wrote the manuscript.

\section{Acknowledgements}

Funding for this work was provided in part by NIHR01CA234969 to Mythreye Karthikeyan (KM) and Nadine Hempel (NH). Balazs Gyorffy (BG) was supported by the FIEK_16-1-2016-0005 and 2020-4.1.1.-TKP2020 grants of the Ministry for Innovation and Technology in Hungary.

We also thank Antonis Kourtidis for generous guidance on the FISH methodology, Jill K. SlackDavis, lain McNeish, Amir Jazaeri and Susan K Murphy for the gift of cell lines.

\section{Conflict of interest}

The authors declare no competing interests.

\section{Figure legends}

Figure 1: PVT1 is amplified in ovarian cancer and impacts patient outcomes.

(A) PVT1 Chromosomal loci copy number gain in ovarian cancer patients from TCGA. (B) Genomic view of chromosome loci 8q24.21. (C) Top 10 correlated genes with MYC (Spearman's correlation). (D) MYC vs PVT1 RNA co-expression in the TCGA ovarian cancer patient datasets (Pearson factor of $0.70-P=3.11$ e-46). (E) PVT1 Copy Number Alterations (CNA) in cancers (top 8) (F) PVT1 RNA expression vs CNA (Pearson factor of 0.46 - $P=4.27$ e-17). (G) Kaplan-Meier analysis for overall survival (log-rank statistics) for ovarian cancer from TCGA. (H) Kaplan-Meier analysis for progression free survival (log-rank statistics) for ovarian cancer from TCGA. (I) Kaplan-Meier analysis for progress free survival by stage (log-rank statistics) for ovarian cancer from TCGA (J) Kaplan-Meier analysis for overall survival by treatment (log-rank statistics) for ovarian cancer from TCGA.

Figure 2: PVT1 levels are altered inn response to changes in cell density.

(A) RT-qPCR analysis of PVT1 levels at baseline in a panel of human OVCA cell lines normalized to non-oncogenic fallopian tube epithelial cell line p211 ( $N=2)(B)$ RT-qPCR analysis of PVT1 levels in indicated cells grown under hypoxia $(0.2 \%)$ normalized to the 
respective levels in normoxia conditions (2way ANOVA-Sidak's multiple comparison test - $\mathrm{n}=3$ - exon 1-2 $P=0.3760$; exon 2-3 $P=0.4203$; exon 6-7 $P=0.0069-\mathrm{N}=3$ ). (C) RT-qPCR analysis of PVT1 RNA expression under anchorage independence $(N=2)$ normalized to D0 (day 0 levels) (D-F) RT-qPCR analysis of PVT1 RNA at different cell densities (inset figures) in indicated cells normalized to the low density levels (2way ANOVA-Sidak's multiple comparison test - exon 1-2 $P<0.0001$; exon 2-3 $P<0.0001$; exon 6-7 $P<0.0001-N=6$ ). (E-F) RT-qPCR analysis of Pvt1 RNA at different cell densities (inset figures) in indicated cells normalized to the low density levels (E) ID8 cells (Unpaired two-tailed t-test - exon 2-3 $P=0.0112-N=3$ ) (F) ID8 Trp53 -/- cells (Unpaired two-tailed t-test - exon 2-3 $P=0.0044-\mathrm{N}=3$ ). (G-H) RT-qPCR analysis of PVT1 RNA in either low or high density of indicated post EMT cells after treatment with TGF- $\beta$ for $96 \mathrm{~h}$ in (G) OVCAR3-M (2way ANOVA-Sidak's multiple comparison test exon 1-2 $P=0.002$; exon 2$3 P=0.0013$; exon 6-7 $P=0.0134-\mathrm{N}=2$ ) and $(\mathrm{H})$ OVCAR420-M (2way ANOVA-Sidak's multiple comparison test - exon 2-3 $P=0.0229$; exon 6-7 $P=0.0086-\mathrm{N}=2$ ). (I) Correlation analysis of PVT1 RNA with gene ontology of epithelial to mesenchymal transition. Error bars are indicated as SEM. Scale bar $=100 \mu \mathrm{m}$. $P$ values are reported as $\geq 0.05$ (ns), 0.01 to $0.05\left(^{*}\right), 0.001$ to $0.01\left(^{* *}\right), 0.0001$ to $0.001\left(^{* * *}\right)$ and $<0.0001\left(^{* * * *}\right)$.

\section{Figure 3: PVT1 is regulated by YAP1.}

(A) Immunofluorescence of YAP1 under low and high density seeding in SK-OV3 cells (scale bar $100 \mu \mathrm{m}$ ). (B) Quantification of the ratio of total YAP to nuclear YAP measured as an average intensity of total YAP in the cell/ total YAP in the nucleus. (Unpaired twotailed t-test - number of cells $=$ minimum 70 cells per biological trial, $P<0.0001-\mathrm{N}=3$ ). (C) RT-qPCR of CTGF and CYR61 mRNA expression of cells grown in indicated cell densities normalized to levels in low density (Unpaired two-tailed t-test - CYR61 $P$ $<0.0001$; CTGF $P=0.0084-\mathrm{N}=3$ ). (D-E) RT-qPCR of (D) PVT1 or (E) indicated genes in cells grown under different matrix rigidity conditions normalized to levels of normal plastic plates (GPa) (2way ANOVA - Tukey's multiple testing $-\mathrm{N}=3$ ). ( $F$ ) RT-qPCR of PVT1 RNA from cells grown at low density and under either full serum or serum starvation as indicated, normalized to levels under full serum (2way ANOVA - Sidak's multiple testing $-\mathrm{N}=2$ - exon 1-2 $P=0.0102$; exon 2-3 $P=0.0068$; exon 6-7 $P=0.0251$ ). (G) RTqPCR of indicated YAP1 target genes of cells grown at low density and under either full serum or serum starvation as indicated, normalized to levels under full serum (2way ANOVA - Sidak's multiple testing - CTGF $P=0.0009 ;$ CYR61 $P=0.0011-\mathrm{N}=2)$. $(\mathbf{H})$ 
RT-qPCR of PVT1 RNA in shRNA YAP1 or ctrl vector cells normalized to levels in Ctrl vector cells (2way ANOVA - Sidak's multiple testing - exon 1-2 $P=0.0199$; YAP1 $P=$ $0.0258-\mathrm{N}=2$ ). (I) RT-qPCR of PVT1 RNA expression in cells treated with $5 \mu \mathrm{M}$ of verteporfin or DMSO control normalized to levels in DMSO treated cells (2way ANOVA Sidak's multiple testing - exon 1-2 $P<0.0001$; exon 2-3 $P=0.0002$; exon 6-7 $P=0.0153$ $-N=3)$. (J) Correlation analysis of PVT1 expression with gene ontology of Hippo signaling pathway. Error bars are indicated as SEM. $P$ values are reported as $\geq 0.05(\mathrm{~ns}), 0.01$ to $\left.0.05{ }^{*}\right), 0.001$ to $\left.0.01{ }^{* *}\right), 0.0001$ to $0.001\left(^{* * *}\right)$ and $<0.0001\left(^{* * * *}\right)$.

Figure 4: PVT1 confers increased migration, invasion and survival advantages to OVCA cells.

(A-B) RT-qPCR of PVT1 in shPVT1 or shCtrl human SK-OV3 and mouse cells (ID8 Trp53 -/-) (Unpaired two-tailed t-test $-P=0.0328$ ) normalized to levels in shCtrl cells. (C) Absorbance values after completion of an MTT assay following $72 \mathrm{~h}$ of growth of shRNA PVT1 or shCtrl SK-OV3 cells (Unpaired two-tailed t-test $-P<0.0001-\mathrm{N}=12$ ). (D) Transwell migration analysis of shRNA PVT1 or shCtrl SK-OV3 cells after $6 \mathrm{~h}$ of migration (Unpaired two-tailed t-test $-P=0.003-N=3$ ). (E) Numbers of colonies counted from a clonogenicity assay using shRNA PVT1 or shCtrl cells after 10 days (Unpaired two-tailed t-test $-P=0.0088-\mathrm{N}=6)(\mathbf{F})$ Absorbance values after completion of a MTT assay after $72 \mathrm{~h}$ using shRNA Pvt1 or shCtrl in mouse ID8 Trp53 -/- cells (Unpaired two-tailed t-test $P<0.0001-\mathrm{N}=12$ ). (G) Transwell migration analysis of shRNA PVT1 or shCtrl mouse ID8 Trp53 -/- cells after $6 \mathrm{~h}$ of migration (Unpaired two-tailed t-test $-P=0.0163-\mathrm{N}=3$ ) (H) RT-qPCR of PVT1 in SK-OV3 cells expressing exogenous pcDNA3.1-PVT1 (Overexpression PVT1) or ctl vector normalized to levels in control cells . (I) RNA-FISH images of cells expressing pcDNA 3.1 - PVT1 exogenously (Overexpression PVT1) or ctl vector in SK-OV3 cells - scale bar $100 \mu \mathrm{m}$. (J) (i) Absorbance values after completion of a MTT assay after $72 \mathrm{~h}$ in indicated cells (Unpaired two-tailed t-test $-P<0.0001-\mathrm{N}=12$ )

(ii) Transwell migration analysis after $6 \mathrm{~h}$ of migration (Unpaired two-tailed t-test $-P=$ $0.0007-\mathrm{N}=3$ ) or (iii) Numbers of colonies counted from a clonogenicity assay in indicated cells (Unpaired two-tailed t-test $-P<0.0001-\mathrm{N}=6$ ). $P$ values are reported as 0.0001 to $\left.0.001{ }^{* * *}\right)$ and $<0.0001\left(^{* * * *}\right)$.

Figure 5: Pvt1 promotes intraperitoneal tumor growth and metastasis in ovarian cancer. 
(A) (i) RT-qPCR of Pvt1 in shPvt1 or shCtrl mouse ID8 Trp53 -/- cells normalized to levels in shctrl cells ( $\mathrm{N}=1$ ) (ii) Representative mouse peritoneal images (above) and (iii) H\&Estained omental tissue sections after 4 weeks following intraperitoneal injection of $5 \times 10^{6}$ shPvt1 or shCtrl mouse ID8 Trp53 -/- cells. Black arrow points to omental tumor/s. Scale bar= $275 \mu \mathrm{m}$. ( $\mathrm{N}=6$ per group) (iv) Weight in grams of the omental tissue at 4 weeks (Unpaired two-tailed t-test $-P=0.0325-\mathrm{N}=6$ ). (B) (i) Representative mouse peritoneal images (above) and (ii) H\&E stained omental tissue sections after 8 weeks following intraperitoneal injection of $5 \times 10^{6}$ shPvt1 or shCtrl mouse ID8 Trp53 -/- cells (iii) Weight in grams of the total tumor burden after 8 weeks (Unpaired two-tailed t-test $-P=0.0113-\mathrm{N}$ = 6) (iv) Volume in $\mathrm{ml}$ of ascites fluid collected from mice after 8 weeks (Unpaired twotailed t-test $-P<0.0001-\mathrm{N}=6)(\mathbf{v})$ Percent change in abdominal girth of mice measured from day 40 to day 60. (Unpaired two-tailed t-test $-P<0.0001-\mathrm{N}=5$ for shCtrl and $\mathrm{n}=6$ for shPvt1).

Figure 6: RNA-sequencing analysis reveals global transcriptional changes with specific changes to genes associated with doxorubicin resistance.

(A) Heat map of the 50 top differentially expressed genes in siPVT1 versus Ctrl vector (sicon) in SK-OV3 cells. (B) gProfiler biological process enrichment analysis in siPVT1 SK-OV3 cells (C) Volcano plot of doxorubicin resistance target genes (AKR1C1, AKR1C2 and AKR1B10) in siPVT1 SK-OV3 cells. (D) GSEA analysis of doxorubicin metabolic process enriched in siPVT1 SK-OV3 cells. (E) RT-qPCR of AKR1C1, AKR1C2 and AKR1B10 in siPVT1 and siCtIr cells (2way ANOVA - Sidak's multiple testing $-P<0.0001$ $-\mathrm{N}=3$ ). (F) IC50 values to doxorubicin (nM) in PVT1 knockdown human SK-OV3 and mouse ID8-IP2 cells as indicated.

\section{Supplementary material}

Figure S1: (A) Kaplan-Meier analysis for progression free survival of grades 2 and 3 (logrank statistics) for ovarian cancer from TCGA. (B) Schematic of PVT1 primer sets and PVT1 shRNAs for human and mouse PVT1 sequence. (C) RT-qPCR analysis of PVT1 RNA at different densities in OV90 cells (2way ANOVA-Sidak's multiple comparison testexon 2-3 $P=0.0005-\mathrm{N}=3$ ) normalized to low density levels (D) RT-qPCR analysis of indicated genes in OVCAR3 and OVCAR420 cells after treatment with 200 pM TGF- $\beta 1$ for $96 \mathrm{~h}$ normalized to vehicle control cells . 
Figure S2: (A) PVT1 expression (RPKM) in sictrl or siPVT1. (B) RT-qPCR analysis of PVT1 in siPVT1 or siCtrl SK-OV3 normalized to values in sicon cells (2way ANOVA Sidak's multiple testing - exon 1-2 $P<0.0001$; exon 2-3 $P<0.0001$; exon 6-7 $<0.0001$ $\mathrm{N}=2$ ). (C) Principal component analysis (PCA) plot of sicon versus siPVT1.

\section{Tables}

Table 1: shRNA sequences

\begin{tabular}{|c|c|}
\hline & shRNA - knockdown \\
\hline Human PVT1 & $\begin{array}{l}\text { Exon 2: GAGCTTCGTTCAAGTATTT } \\
\text { Exon 8: GAAATGTCCTCTCGCCTGC }\end{array}$ \\
\hline Human control & SMARTvector Non-targeting hCMV-TurboRFP plasmid \\
\hline Mouse pvt1 & $\begin{array}{l}\text { Exon 1: CGAGTGTGAAGGAGCGAGT } \\
\text { Exon 4: TGACCTTATTGTAGACTAA }\end{array}$ \\
\hline Mouse control & pHIV-Zsgreen plasmid \\
\hline Human YAP1 & $\begin{array}{l}\text { CCGGCCCAGTTAAATGTTCACCAATCTCGAGATTGGTGAACATTTAAC } \\
\text { TGGGTTTTTG } \\
\text { CCGGGACCAATAGCTCAGATCCTTTCTCGAGAAAGGATCTGAGCTATT } \\
\text { GGTCTTTTTG }\end{array}$ \\
\hline Human control & TRCN scr PLKO.1 plasmid \\
\hline
\end{tabular}

Table 2: Primer sequences

\begin{tabular}{|l|l|l|}
\hline Gene ID & Primer sequence (5'->3') & Specie \\
\hline RPL13a & Forw: AGATGGCGGAGGTGCAG & Human \\
\hline PVT1 exon 1-2 & $\begin{array}{l}\text { Forw: CACCTTCCAGTGGATTTCCTT Rev: } \\
\text { GACAGGCACAGCCATCTT }\end{array}$ & \\
\hline
\end{tabular}




\begin{tabular}{|c|c|c|}
\hline PVT1 exon 2-3 & $\begin{array}{l}\text { Forw: CTTCCTGGTGAAGCATCTGAT } \\
\text { Rev: TTCAGCCTCCACTTAAAGTACC }\end{array}$ & \\
\hline PVT1 exon 6-7 & $\begin{array}{l}\text { Forw: CTGTTTGCTTCTCCTGTTGC } \\
\text { Rev: GAACTCCTCAGCCTCCAAG }\end{array}$ & \\
\hline CTGF & $\begin{array}{l}\text { Forw: GCGTGTGCACCGCCAAAGAT } \\
\text { Rev: CAGGGCTGGGCAGACGAACG }\end{array}$ & \\
\hline CYR61 & $\begin{array}{l}\text { Forw: CGCCTTGTGAAAGAAACCCG } \\
\text { Rev: GGTTCGGGGGATTTCTTGGT }\end{array}$ & \\
\hline YAP1 & $\begin{array}{l}\text { Forw: TGACCCTCGTTTTGCCATGA } \\
\text { Rev: GTTGCTGCTGGTTGGAGTTG }\end{array}$ & \\
\hline SNAIL 1 & $\begin{array}{l}\text { Forw: AAGATGCACATCCGAAGCCA } \\
\text { Rev: CAGTGGGAGCAGGAGAATGG }\end{array}$ & \\
\hline ZEB1 & $\begin{array}{l}\text { Forw: CTGCTCCCTGTGCAGTTACA } \\
\text { Rev: GTGCACTTGAACTTGCGGTT }\end{array}$ & \\
\hline \multirow[t]{2}{*}{ AKR1C1 } & Forw: GTCCTGGCCAAGAGCTACAA & \\
\hline & Rev:CGCACATTTCTGTTTAGGCCAT & \\
\hline \multirow[t]{2}{*}{ AKR1C2 } & Forw: ACGGAGTCATTGCCATTCAGA & \\
\hline & Rev:CATGCAATGCCCTCCATGTTA & \\
\hline \multirow[t]{2}{*}{ AKR1B10 } & Forw: AGAAACTGGAGGGCCTGTAA & \\
\hline & Rev:GGAGATTCAACCTCAATATTTGC & \\
\hline Rpl13a & $\begin{array}{l}\text { Forw: CAAGGTTGTTCGGCTGAAGC } \\
\text { Rev: GCTGTCACTGCCTGGTACTT }\end{array}$ & Mouse \\
\hline Pvt1 exon 2-3 & $\begin{array}{l}\text { Forw: CACTGAAAACAAGGACCGAAAC Rev: } \\
\text { ACAGACATTGGCAGTGGC }\end{array}$ & \\
\hline
\end{tabular}




\section{References}

1. Hanahan, D. and R.A. Weinberg, Hallmarks of cancer: the next generation. Cell, 2011. 144(5): p. 646-74.

2. Siegel, R.L., K.D. Miller, and A. Jemal, Cancer statistics, 2020. CA: A Cancer Journal for Clinicians, 2020. 70(1): p. 7-30.

3. Reid, B.M., J.B. Permuth, and T.A. Sellers, Epidemiology of ovarian cancer: a review. Cancer biology \& medicine, 2017. 14(1): p. 9-32.

4. $\quad$ Lheureux, S., et al., Epithelial ovarian cancer. Lancet, 2019. 393(10177): p. 1240-1253.

5. Karnezis, A.N., et al., The disparate origins of ovarian cancers: pathogenesis and prevention strategies. Nature Reviews Cancer, 2017. 17(1): p. 65-74.

6. $\quad$ Kumari, A., et al., TGF $\beta$ signaling networks in ovarian cancer progression and plasticity. Clin Exp Metastasis, 2021. 38(2): p. 139-161.

7. Klemba, A., et al., Hypoxia-Mediated Decrease of Ovarian Cancer Cells Reaction to Treatment: Significance for Chemo- and Immunotherapies. Int J Mol Sci, 2020. 21(24).

8. Rankin, E.B., J.-M. Nam, and A.J. Giaccia, Hypoxia: Signaling the Metastatic Cascade. Trends in Cancer, 2016. 2(6): p. 295-304.

9. $\quad \mathrm{Xu}, \mathrm{W}$. , et al., Cell Stiffness Is a Biomarker of the Metastatic Potential of Ovarian Cancer Cells. PLOS ONE, 2012. 7(10): p. e46609.

10. Swaminathan, V., et al., Mechanical stiffness grades metastatic potential in patient tumor cells and in cancer cell lines. Cancer Res, 2011. 71(15): p. 5075-80.

11. McKenzie, A.J., et al., The mechanical microenvironment regulates ovarian cancer cell morphology, migration, and spheroid disaggregation. Scientific Reports, 2018. 8(1): p. 7228.

12. Lander, E.S., Initial impact of the sequencing of the human genome. Nature, 2011. 470(7333): p. 187-197.

13. Rupaimoole, R. and F.J. Slack, MicroRNA therapeutics: towards a new era for the management of cancer and other diseases. Nature Reviews Drug Discovery, 2017. 16(3): p. 203-222.

14. Ozata, D.M., et al., PIWI-interacting RNAs: small RNAs with big functions. Nature Reviews Genetics, 2019. 20(2): p. 89-108.

15. Oncul, S., et al., Long non-coding RNAs in ovarian cancer: expression profile and functional spectrum. RNA Biology, 2019: p. 1-12.

16. Guan, Y., et al., Amplification of PVT1 Contributes to the Pathophysiology of Ovarian and Breast Cancer. Clinical Cancer Research, 2007. 13(19): p. 5745-5755.

17. Tseng, Y.-Y., et al., PVT1 dependence in cancer with MYC copy-number increase. Nature, 2014. 512(7512): p. 82-86. 
18. Wang, W., et al., PVT1 Promotes Cancer Progression via MicroRNAs. Frontiers in Oncology, 2019. 9(609).

19. Li, T., X.-1. Meng, and W.-q. Yang, Long Noncoding RNA PVT1 Acts as a "Sponge” to Inhibit microRNA-152 in Gastric Cancer Cells. Digestive Diseases and Sciences, 2017. 62(11): p. 3021-3028.

20. Liu, E., Z. Liu, and Y. Zhou, Carboplatin-docetaxel-induced activity against ovarian cancer is dependent on up-regulated lncRNA PVT1. International journal of clinical and experimental pathology, 2015. 8(4): p. 3803-3810.

21. Yi, K., et al., LncRNA PVT1 epigenetically stabilizes and post-transcriptionally regulates FOXM1 by acting as a microRNA sponge and thus promotes malignant behaviors of ovarian cancer cells. American journal of translational research, 2020. 12(6): p. 28602874.

22. Salamini-Montemurri, M., et al., The Challenges and Opportunities of LncRNAs in Ovarian Cancer Research and Clinical Use. Cancers, 2020. 12(4): p. 1020.

23. Cerami, E., et al., The cBio cancer genomics portal: an open platform for exploring multidimensional cancer genomics data. Cancer Discov, 2012. 2(5): p. 401-4.

24. Gao, J., et al., Integrative analysis of complex cancer genomics and clinical profiles using the cBioPortal. Sci Signal, 2013. 6(269): p. pl1.

25. Bell, D., et al., Integrated genomic analyses of ovarian carcinoma. Nature, 2011. 474(7353): p. 609-615.

26. Gyorffy, B., A. Lánczky, and Z. Szállási, Implementing an online tool for genome-wide validation of survival-associated biomarkers in ovarian-cancer using microarray data from 1287 patients. Endocr Relat Cancer, 2012. 19(2): p. 197-208.

27. Nagy, Á., et al., Validation of miRNA prognostic power in hepatocellular carcinoma using expression data of independent datasets. Scientific Reports, 2018. 8(1): p. 9227.

28. Yang, Q., et al., Long non-coding RNA PVT1 promotes cell proliferation and invasion through regulating miR-133a in ovarian cancer. Biomedicine \& pharmacotherapy $=$ Biomedecine \& pharmacotherapie, 2018. 106: p. 61-67.

29. Sun, L., et al., MicroRNA-149 suppresses the proliferation and increases the sensitivity of ovarian cancer cells to cisplatin by targeting X-linked inhibitor of apoptosis. Oncology letters, 2018. 15(5): p. 7328-7334.

30. Yi, B.R., et al., Alteration of epithelial-mesenchymal transition markers in human normal ovaries and neoplastic ovarian cancers. Int J Oncol, 2015. 46(1): p. 272-80.

31. Kim, W., et al., Hippo signaling is intrinsically regulated during cell cycle progression by $A P C / C^{C d h 1}$. Proceedings of the National Academy of Sciences, 2019. 116(19): p. 9423.

32. Dobrokhotov, O., et al., Mechanoregulation and pathology of YAP/TAZ via Hippo and non-Hippo mechanisms. Clin Transl Med, 2018. 7(1): p. 23.

33. Piccolo, S., S. Dupont, and M. Cordenonsi, The Biology of YAP/TAZ: Hippo Signaling and Beyond. Physiological Reviews, 2014. 94(4): p. 1287-1312.

34. Yu, F.X., et al., Regulation of the Hippo-YAP pathway by G-protein-coupled receptor signaling. Cell, 2012. 150(4): p. 780-91.

35. Liu-Chittenden, Y., et al., Genetic and pharmacological disruption of the TEAD-YAP complex suppresses the oncogenic activity of YAP. Genes Dev, 2012. 26(12): p. 1300-5.

36. Noviello, T.M.R., et al., Detection of long non-coding RNA homology, a comparative study on alignment and alignment-free metrics. BMC Bioinformatics, 2018. 19(1): p. 407. 
37. Zampetaki, A., A. Albrecht, and K. Steinhofel, Long Non-coding RNA Structure and Function: Is There a Link? Frontiers in Physiology, 2018. 9(1201).

38. Cai, Q., L. Yan, and Y. Xu, Anoikis resistance is a critical feature of highly aggressive ovarian cancer cells. Oncogene, 2015. 34(25): p. 3315-24.

39. Yaginuma, Y. and H. Westphal, Abnormal structure and expression of the p53 gene in human ovarian carcinoma cell lines. Cancer Res, 1992. 52(15): p. 4196-9.

40. $\quad$ Nakayama, J., et al., Decreased peritoneal ovarian cancer growth in mice lacking expression of lipid phosphate phosphohydrolase 1. PLoS One, 2015. 10(3): p. e0120071.

41. Jin, K., et al., Long non-coding RNA PVT1 interacts with MYC and its downstream molecules to synergistically promote tumorigenesis. Cellular and molecular life sciences : CMLS, 2019. 76(21): p. 4275-4289.

42. Cho, S.W., et al., Promoter of IncRNA Gene PVT1 Is a Tumor-Suppressor DNA Boundary Element. Cell, 2018. 173(6): p. 1398-1412.e22.

43. Sud, A., B. Kinnersley, and R.S. Houlston, Genome-wide association studies of cancer: current insights and future perspectives. Nature Reviews Cancer, 2017. 17(11): p. 692704.

44. Goode, E.L., et al., A genome-wide association study identifies susceptibility loci for ovarian cancer at $2 q 31$ and 8q24. Nat Genet, 2010. 42(10): p. 874-9.

45. Phelan, C.M., et al., Identification of 12 new susceptibility loci for different histotypes of epithelial ovarian cancer. Nature Genetics, 2017. 49(5): p. 680-691.

46. $\quad$ Dang, Chi V., MYC on the Path to Cancer. Cell, 2012. 149(1): p. 22-35.

47. Ding, Y., et al., Amplification of IncRNA PVT1 promotes ovarian cancer proliferation by binding to miR-140. Mammalian Genome, 2019. 30(7): p. 217-225.

48. Chen, Y., et al., LncRNA PVT1 promotes ovarian cancer progression by silencing miR214. Cancer biology \& medicine, 2018. 15(3): p. 238-250.

49. Iden, M., et al., The IncRNA PVT1 Contributes to the Cervical Cancer Phenotype and Associates with Poor Patient Prognosis. PLOS ONE, 2016. 11(5): p. e0156274.

50. Yu, F., et al., Hypoxia induces the activation of hepatic stellate cells through the PVT1miR-152-ATG14 signaling pathway. Molecular and Cellular Biochemistry, 2020. 465(1): p. 115-123.

51. Wang, C., et al., LncRNA PVT1 regulate expression of HIF $1 \alpha$ via functioning as ceRNA for miR-199a-5p in non-small cell lung cancer under hypoxia. Mol Med Rep, 2018. 17(1): p. 1105-1110.

52. Zhang, X., et al., The Hippo pathway transcriptional co-activator, YAP, is an ovarian cancer oncogene. Oncogene, 2011. 30(25): p. 2810-22.

53. Xia, Y., et al. YAP promotes ovarian cancer cell tumorigenesis and is indicative of a poor prognosis for ovarian cancer patients. PloS one, 2014. 9, e91770 DOI: 10.1371/journal.pone.0091770.

54. Han, Y., Analysis of the role of the Hippo pathway in cancer. Journal of Translational Medicine, 2019. 17(1): p. 116.

55. Huang, R.Y., et al., An EMT spectrum defines an anoikis-resistant and spheroidogenic intermediate mesenchymal state that is sensitive to e-cadherin restoration by a src-kinase inhibitor, saracatinib (AZD0530). Cell Death Dis, 2013. 4(11): p. e915.

56. Zanconato, F., M. Cordenonsi, and S. Piccolo, YAP/TAZ at the Roots of Cancer. Cancer Cell, 2016. 29(6): p. 783-803. 
57. Grannas, K., et al., Crosstalk between Hippo and TGF 8 : Subcellular Localization of YAP/TAZ/Smad Complexes. Journal of Molecular Biology, 2015. 427(21): p. 3407-3415.

58. $\quad$ Verduci, L., et al., The oncogenic role of circPVT1 in head and neck squamous cell carcinoma is mediated through the mutant p53/YAP/TEAD transcription-competent complex. Genome Biology, 2017. 18(1): p. 237.

59. Xu, M.-d., et al., A Positive Feedback Loop of IncRNA-PVT1 and FOXM1 Facilitates Gastric Cancer Growth and Invasion. Clinical Cancer Research, 2017. 23(8): p. 2071.

60. Zhao, J., et al., LncRNA PVT1 promotes angiogenesis via activating the STAT3/VEGFA axis in gastric cancer. Oncogene, 2018. 37(30): p. 4094-4109.

61. Olivero, C.E., et al., p53 Activates the Long Noncoding RNA Pvt1b to Inhibit Myc and Suppress Tumorigenesis. Mol Cell, 2020. 77(4): p. 761-774 e8.

62. Fan, Q., Q. Cai, and Y. Xu, FOXM1 is a downstream target of LPA and YAP oncogenic signaling pathways in high grade serous ovarian cancer. Oncotarget; Vol 6, No 29, 2015.

63. Barsotti, A.M., et al., p53-Dependent induction of PVT1 and miR-1204. The Journal of biological chemistry, 2012. 287(4): p. 2509-2519.

64. Zeng, C.-M., et al., Aldo-Keto Reductase AKR1C1-AKR1C4: Functions, Regulation, and Intervention for Anti-cancer Therapy. Frontiers in Pharmacology, 2017. 8(119).

65. Matsunaga, T., et al., Aldo-Keto Reductase 1 B10 and Its Role in Proliferation Capacity of Drug-Resistant Cancers. Front Pharmacol, 2012. 3: p. 5.

66. Heibein, A.D., et al., Role of aldo-keto reductases and other doxorubicin pharmacokinetic genes in doxorubicin resistance, DNA binding, and subcellular localization. BMC Cancer, 2012. 12(1): p. 381.

67. DiStefano, J.K. and B. Davis, Diagnostic and Prognostic Potential of AKR1B10 in Human Hepatocellular Carcinoma. Cancers (Basel), 2019. 11(4).

68. Zhang, S., et al., Identification of the molecular basis of doxorubicin-induced cardiotoxicity. Nat Med, 2012. 18(11): p. 1639-42.

69. Arun, G., S.D. Diermeier, and D.L. Spector, Therapeutic Targeting of Long Non-Coding RNAs in Cancer. Trends Mol Med, 2018. 24(3): p. 257-277.

70. Varadaraj, A., et al., Epigenetic Regulation of GDF2 Suppresses Anoikis in Ovarian and Breast Epithelia. Neoplasia, 2015. 17(11): p. 826-38.

71. Jazaeri, A.A., et al., Molecular requirements for transformation of fallopian tube epithelial cells into serous carcinoma. Neoplasia, 2011. 13(10): p. 899-911.

72. Mi, H., et al., PANTHER version 14: more genomes, a new PANTHER GO-slim and improvements in enrichment analysis tools. Nucleic Acids Res, 2019. 47(D1): p. D419d426.

73. Vichai, V. and K. Kirtikara, Sulforhodamine B colorimetric assay for cytotoxicity screening. Nat Protoc, 2006. 1(3): p. 1112-6.

74. Dobin, A., et al., STAR: ultrafast universal RNA-seq aligner. Bioinformatics, 2013. 29(1): p. 15-21.

75. Patro, R., et al., Salmon provides fast and bias-aware quantification of transcript expression. Nat Methods, 2017. 14(4): p. 417-419.

76. Love, M.I., W. Huber, and S. Anders, Moderated estimation of fold change and dispersion for RNA-seq data with DESeq2. Genome Biology, 2014. 15(12): p. 550. 
bioRxiv preprint doi: https://doi.org/10.1101/2022.01.11.475893; this version posted January 13,2022 . The copyright holder for this preprint

(which was not certified by peer review) is the author/funder, who has granted bioRxiv a license to display the preprint in perpetuity. It is made available under aCC-BY-NC-ND 4.0 International license.

77. Subramanian, A., et al., Gene set enrichment analysis: A knowledge-based approach for interpreting genome-wide expression profiles. Proceedings of the National Academy of Sciences, 2005. 102(43): p. 15545.

78. Mootha, V.K., et al., PGC-1 $\alpha$-responsive genes involved in oxidative phosphorylation are coordinately downregulated in human diabetes. Nature Genetics, 2003. 34(3): p. 267273. 
Fig bldF v preprint doi: https://doi.org/10.1101/2022.01.11.475893; this version posted January 13, 2022. The copyright holder for this preprint (which was not certified by peer review) is the author/funder, who has granted bioRxiv a license to display the preprint in perpetuity. It is made A available un $\mathrm{B}$ aCC-BY-NC-ND 4.0 International license.
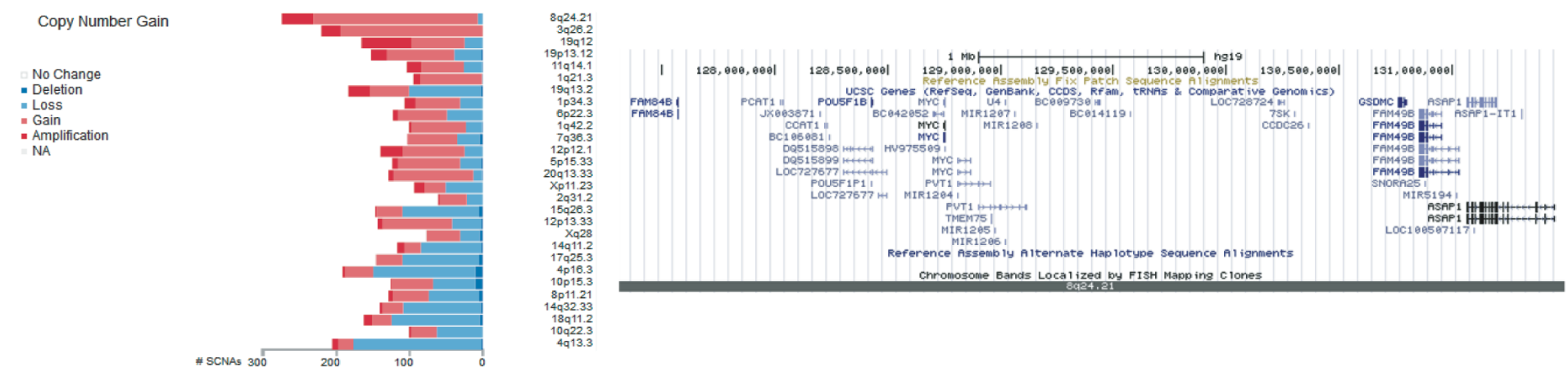

C

\begin{tabular}{cccc|}
\hline $\begin{array}{c}\text { Correlated } \\
\text { Gene }\end{array}$ & $\begin{array}{c}\text { Spearman's } \\
\text { Correlation }\end{array}$ & p-Value & q-Value \\
\hline PVT1 & 0.704 & $3.27 \mathrm{e}-47$ & $6.57 \mathrm{e}-43$ \\
EIF4EBP3 & 0.470 & $2.80 \mathrm{e}-18$ & $1.94 \mathrm{e}-14$ \\
CGREF1 & 0.470 & $2.89 \mathrm{e}-18$ & $1.94 \mathrm{e}-14$ \\
LONRF2 & 0.445 & $2.43 \mathrm{e}-16$ & $1.12 \mathrm{e}-12$ \\
EEF1G & 0.444 & $2.78 \mathrm{e}-16$ & $1.12 \mathrm{e}-12$ \\
RPL4 & 0.441 & $4.49 \mathrm{e}-16$ & $1.43 \mathrm{e}-12$ \\
ACTR1A & -0.440 & $5.37 \mathrm{e}-16$ & $1.43 \mathrm{e}-12$ \\
CDC42SE1 & -0.440 & $5.99 \mathrm{e}-16$ & $1.43 \mathrm{e}-12$ \\
INA & -0.439 & $6.41 \mathrm{e}-16$ & $1.43 \mathrm{e}-12$ \\
LARS & 0.432 & $2.13 \mathrm{e}-15$ & $4.30 \mathrm{e}-12$ \\
BMF & -0.426 & $5.40 \mathrm{e}-15$ & $8.81 \mathrm{e}-12$ \\
\hline
\end{tabular}

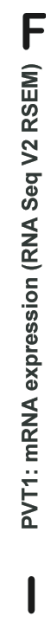

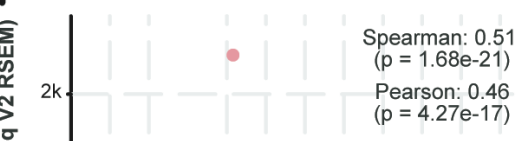

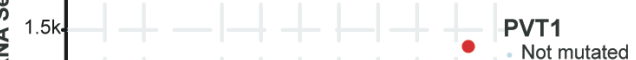

$1 \mathrm{k}$ - 1 - Amplification

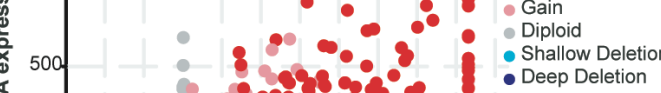

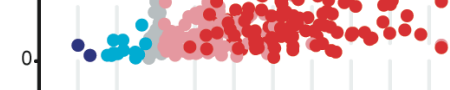

$\begin{array}{llllllllllllll}-1 & -0.5 & 0 & 0.5 & 1 & 1.5 & 2 & 2.5 & 3 & 3.5 & 4\end{array}$

PVT1: Capped relative linear copy-number values

Progression free survival_stage 2

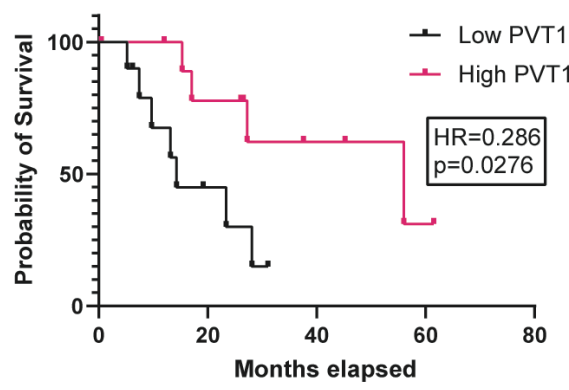

$\checkmark$

Overall survival_any chemo

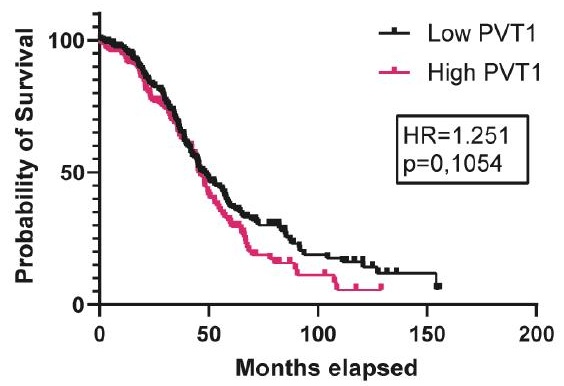

D氶

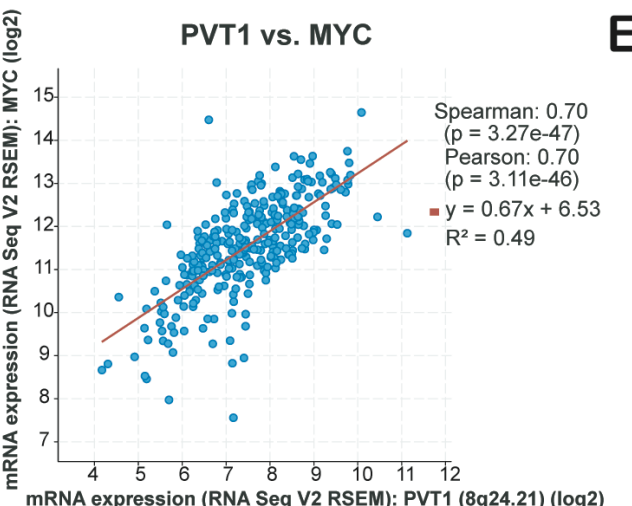

G

$\mathrm{H}$

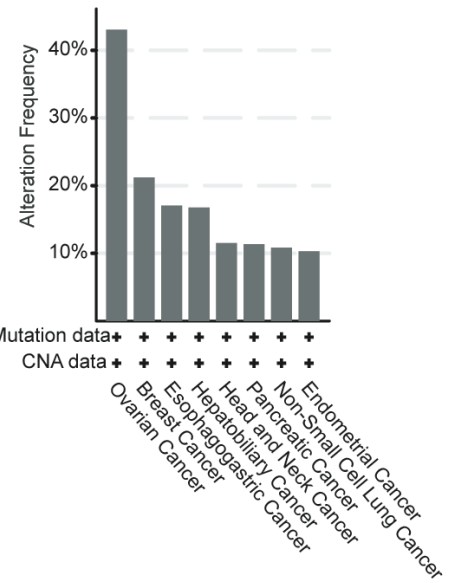

Progression free survival_all
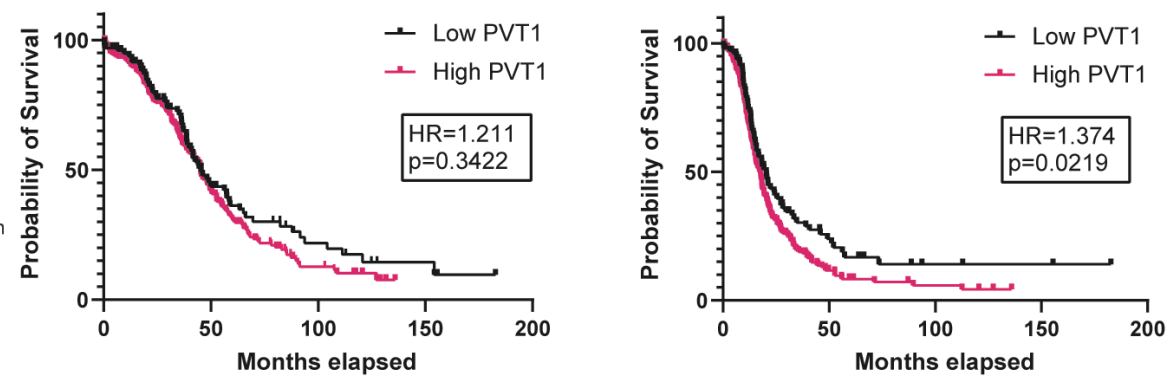

Progression free survival_stage 3

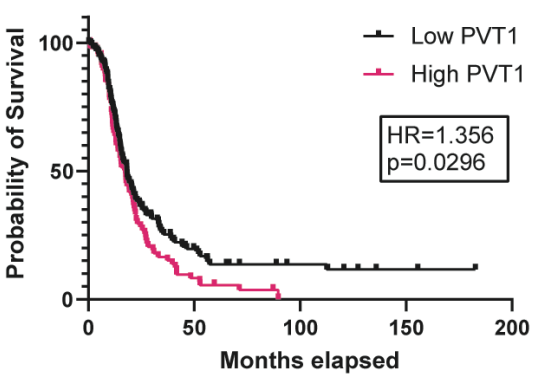

Progression free survival_stage 4

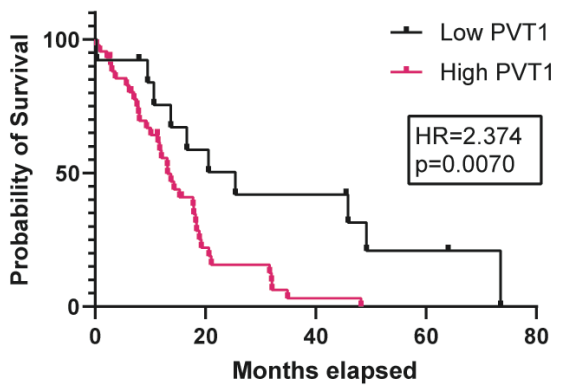

Overall survival_targeted therapy

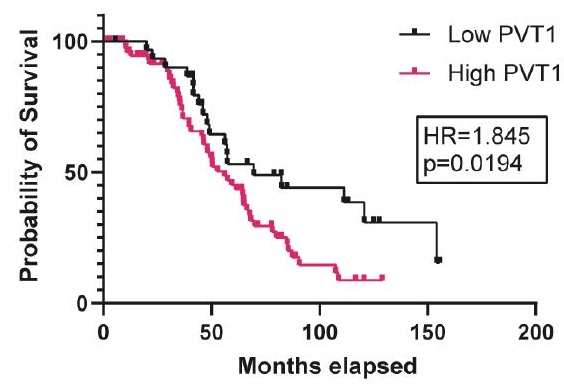


FigldRe Peprint doi: https://doi.org/10.1101/2022.01.11.475893; this version posted January 13, 2022. The copyright holder for this preprint A (which was not certified by peer review) is the author/funder, who has granted biokxiv a license to display the preprint in perpetuity. It is made A PVT1 baseline level available under aCC-BY-NC-ND 4.0 In Brnational lsieres Hypoxia
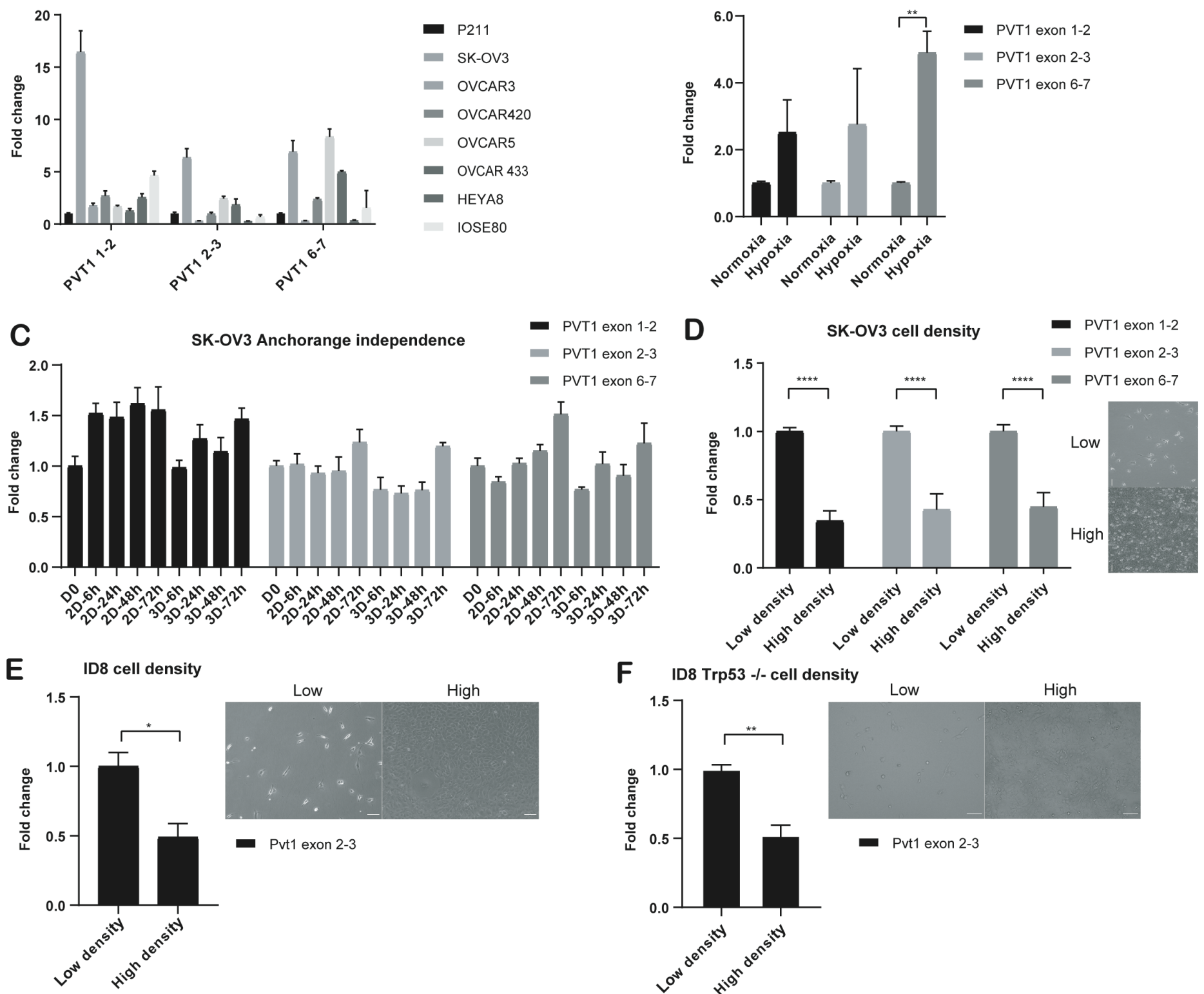

- Pvt1 exon 2-3

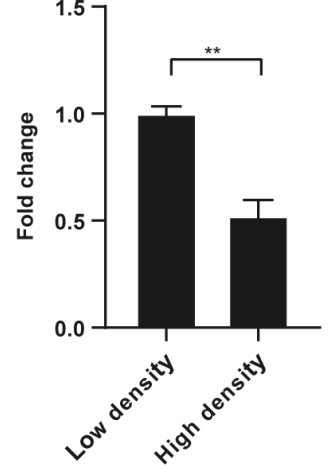

High

G

OVCAR3-M

H

OVCAR420-M
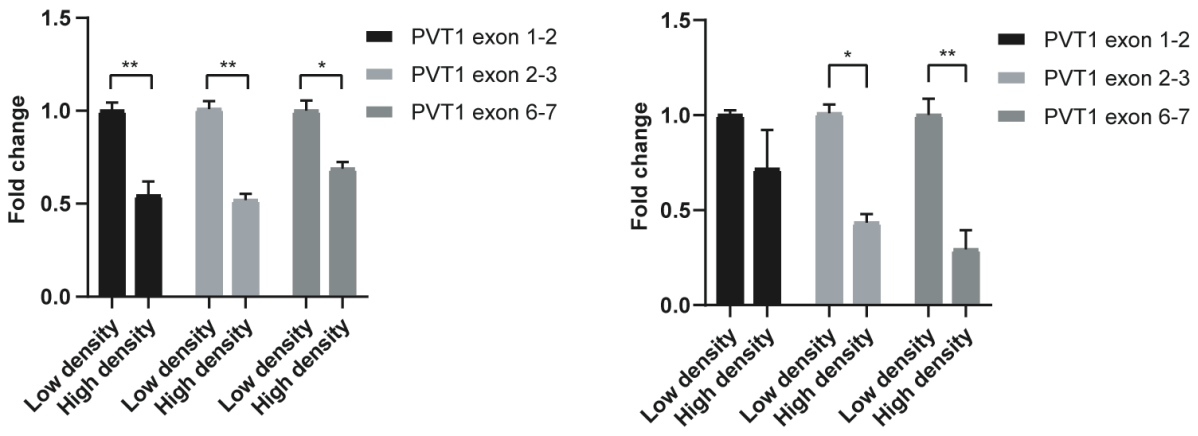

I GO Epithelial to Mesenchymal transition
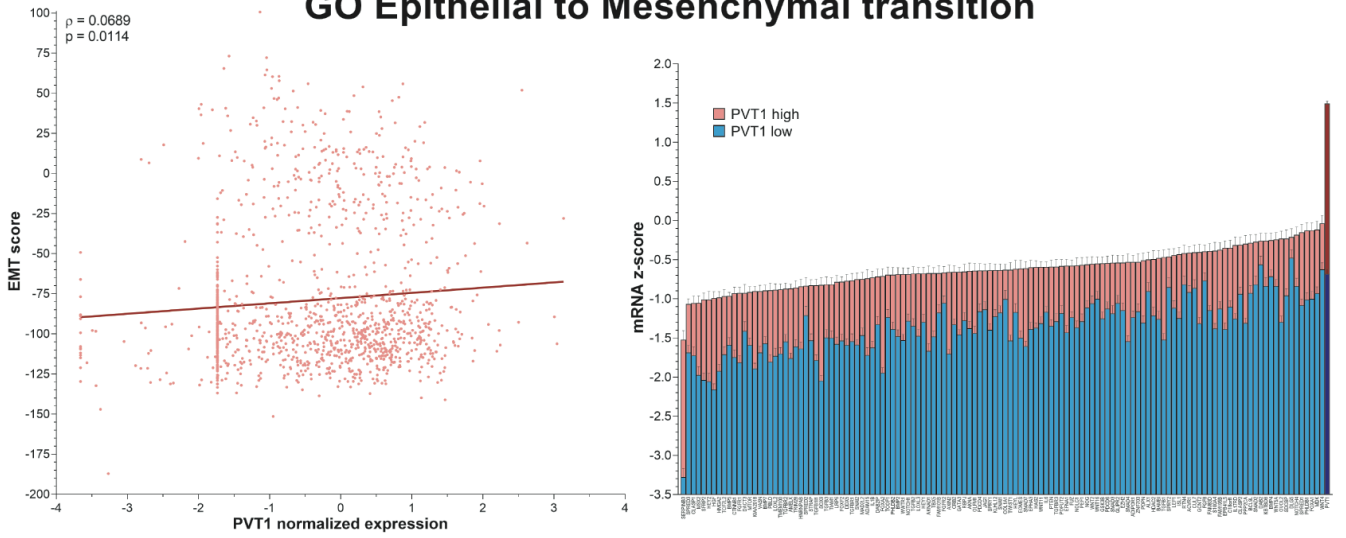
Figkkreræ3rint doi: https://doi.org/10.1101/2022.01.11.475893; this version posted January 13, 2022. The copyright holder for this preprint Awhich was not certified by peer review) is the author/funde $\mathbf{B}$ who has granted bioRxiv a lice (se to display the preprint in perpetuity. It is made
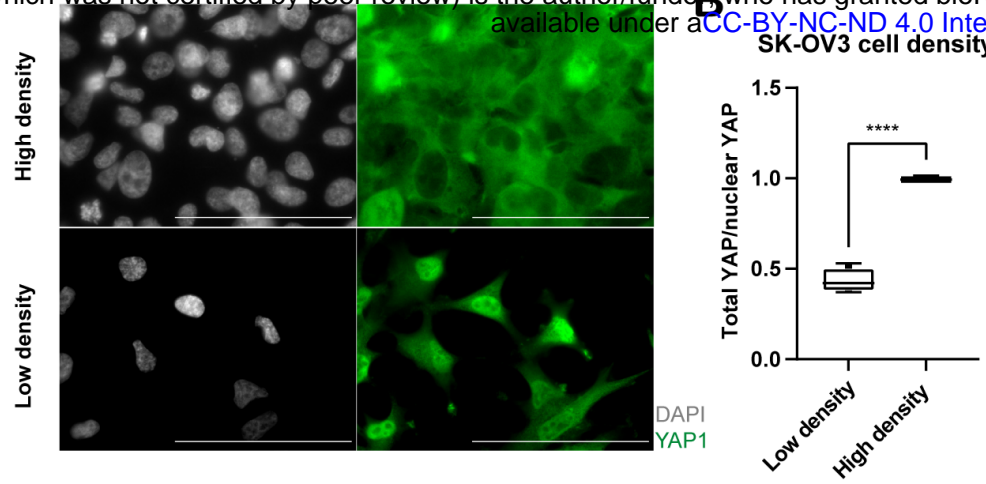

SK-OV3 matrix rigidity

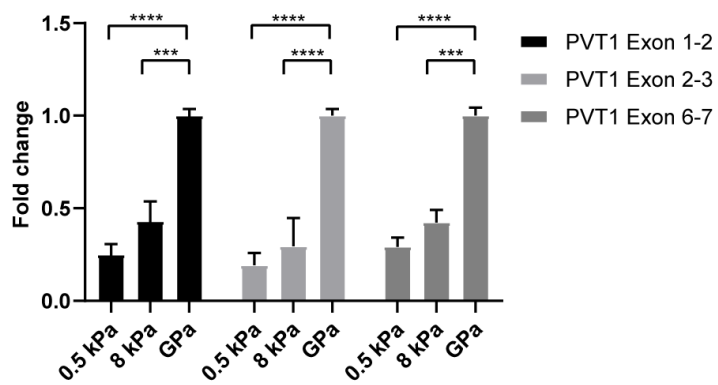

$\mathbf{F}$ SK-OV3

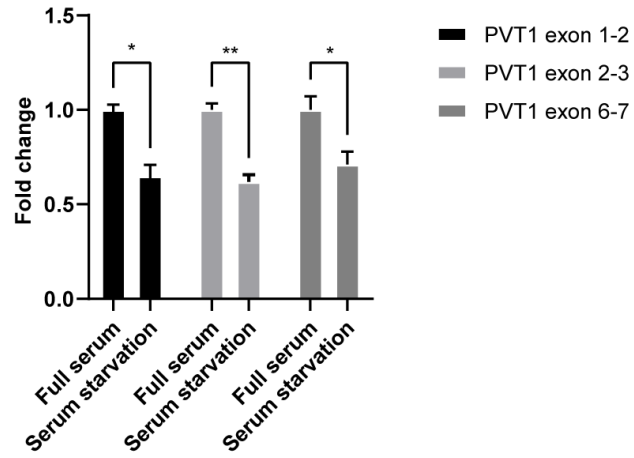

H

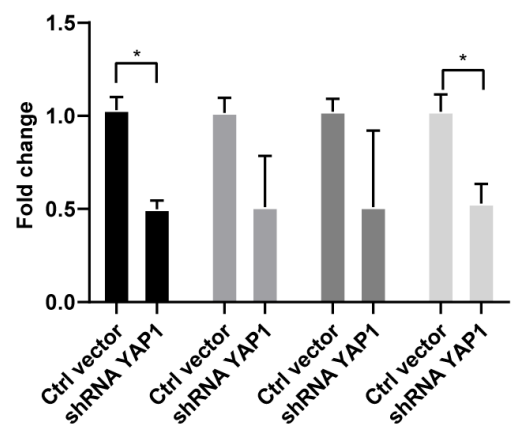

- PVT1 exon 1-2

- PVT1 exon 2-3

- PVT1 exon 6-7

- YAP1

$J$
SK-OV3 cell density

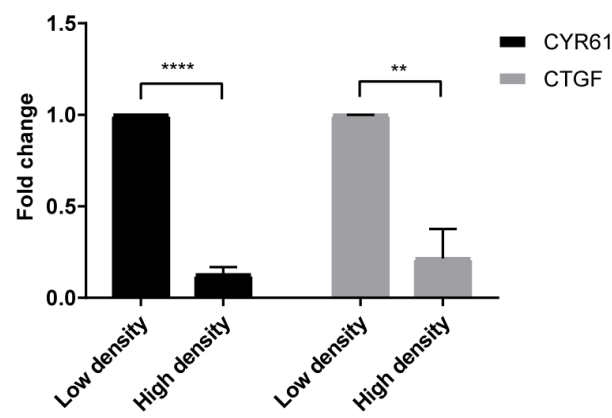

E

G
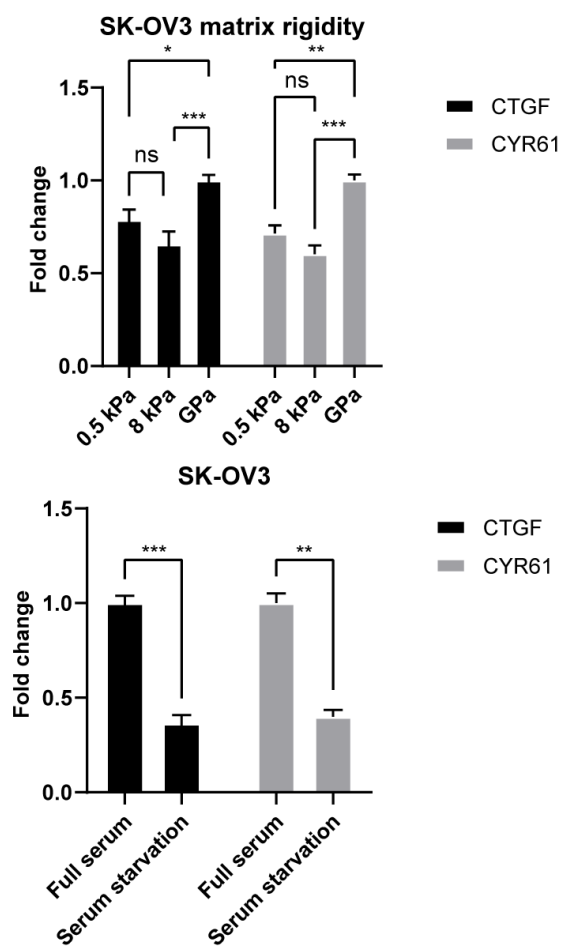

- CTGF

- CYR61

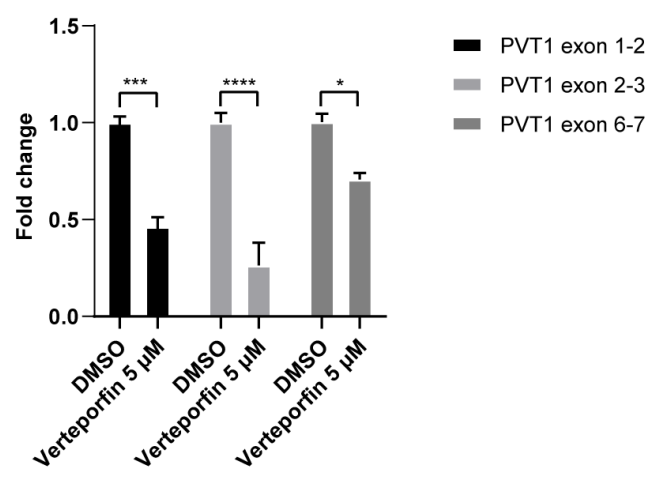

GO Hippo signaling

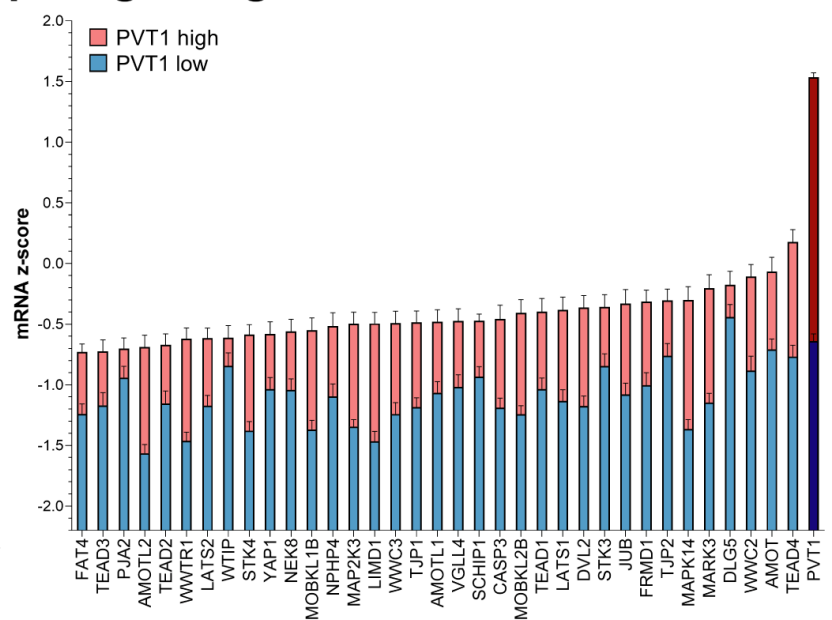


FigdakkepAprint doi: https://doi.org/10.1101/2022.01.11.475893; this version posted January 13, 2022. The copyright holder for this preprint (which was not certified by peex review) is the author/funder, who has granted bioRxiv a license to display the preprint in perpetuity. It is made available under aCC-BY-NC-ND 4.0 InteBational license.
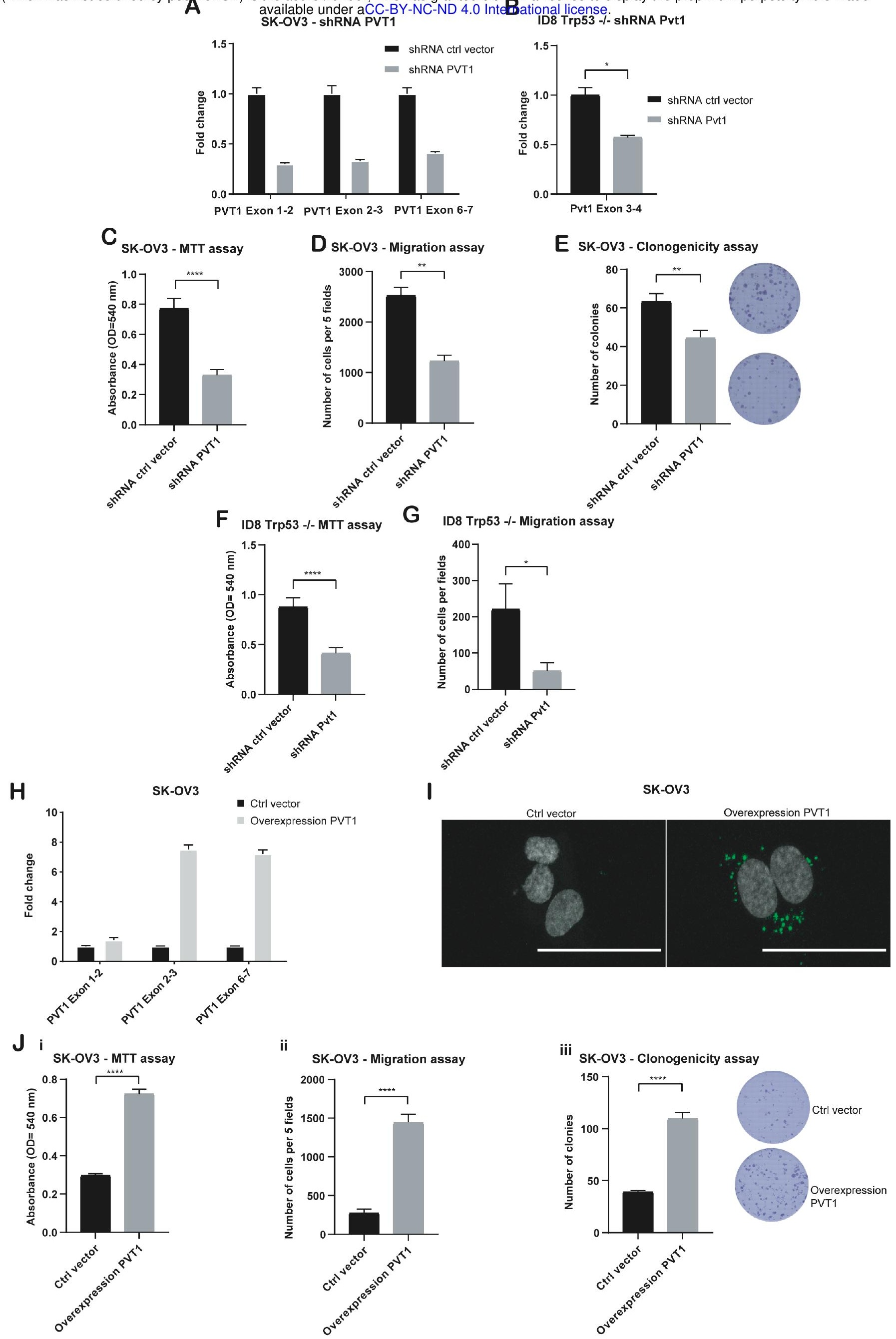
bioRxiv preprint doi: https://doi.org/10.1101/2022.01.11.475893; this version posted January 13,2022 . The copyright holder for this preprint (which was not certified by peer review) is the author/funder, who has granted bioRxiv a license to display the preprint in perpetuity. It is made available under aCC-BY-NC-ND 4.0 International license.

\section{Figure 5}

A

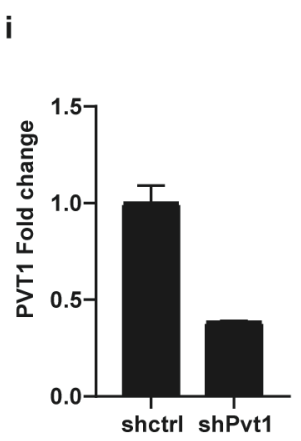

B

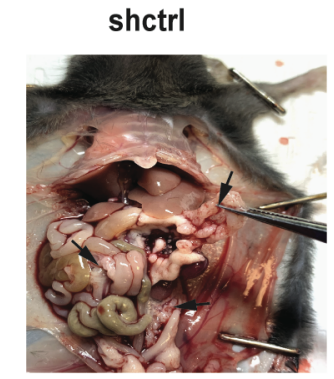

ii

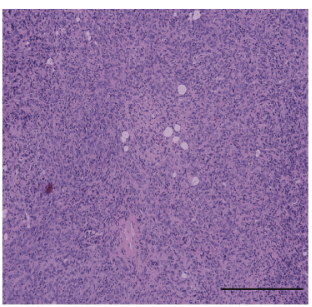

4 Weeks

ii

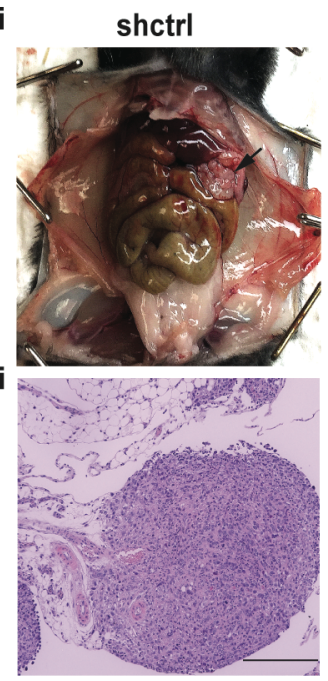

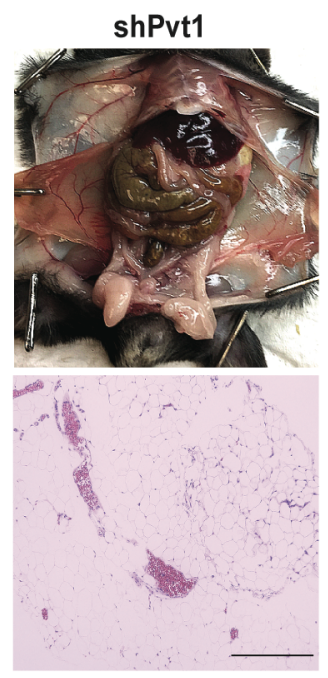

iv

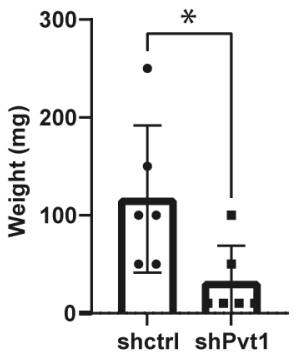

8 Weeks

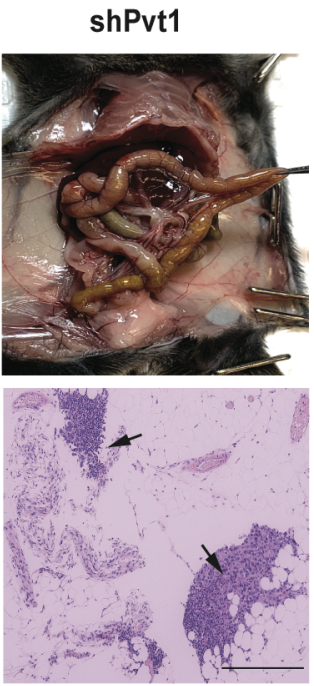

iii

Tumor burden (8wk)

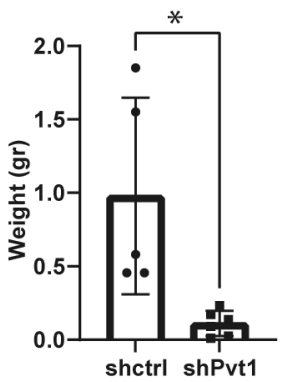

iv

Ascites fluid

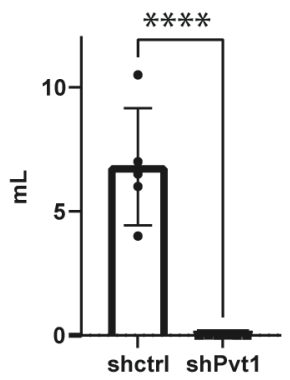

v

Abdominal girth

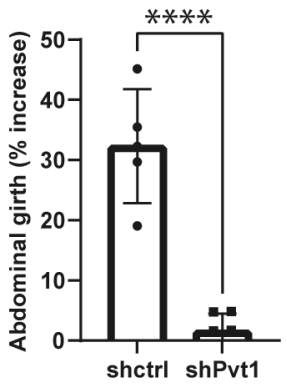


Figure GoRxiv preprint doi: https://doi.org/10.1101/2022.01.11.475893; this version posted January 13 , 2022. The copyright holder for this preprint A (which was not certified by peer review) is the author/funder, who has granted bioRxiv a license to display the preprint in perpetuity. It is made available under aCC-BY-NC-ND 4.0 International license.

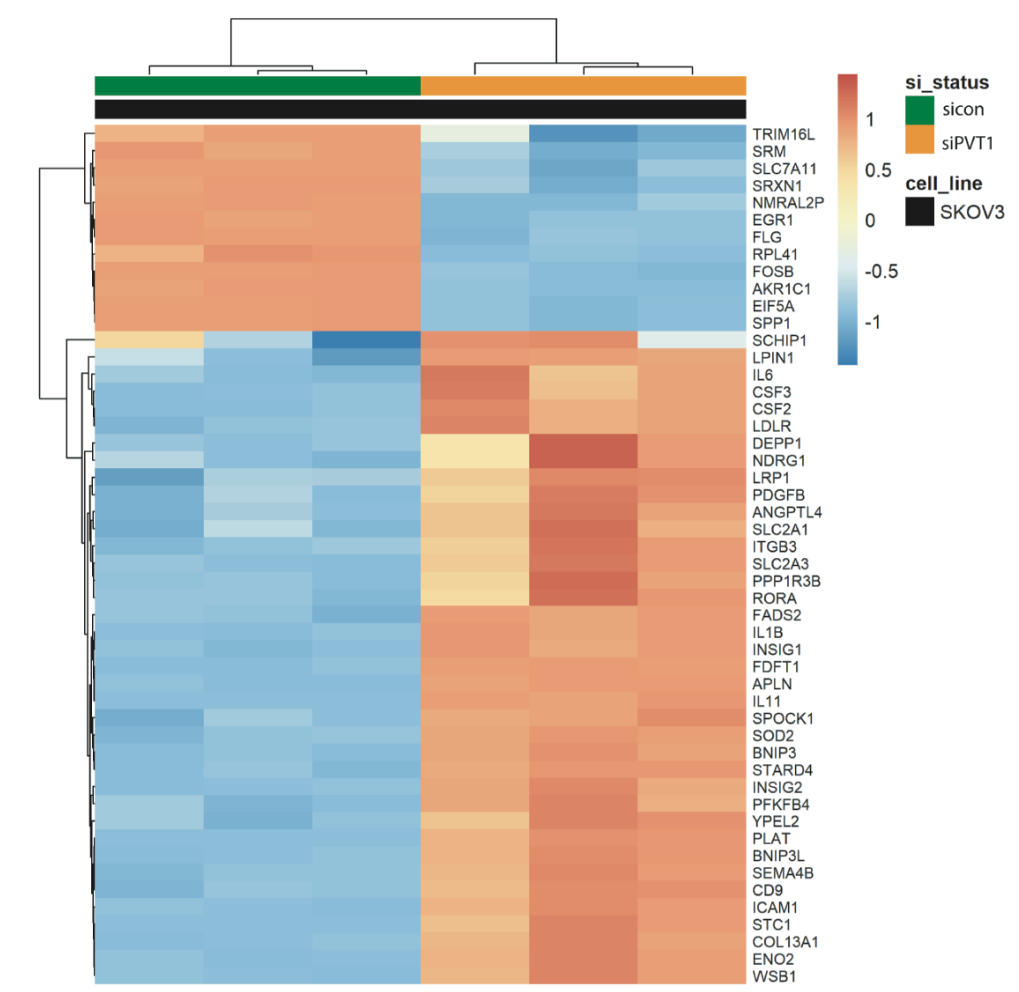

B
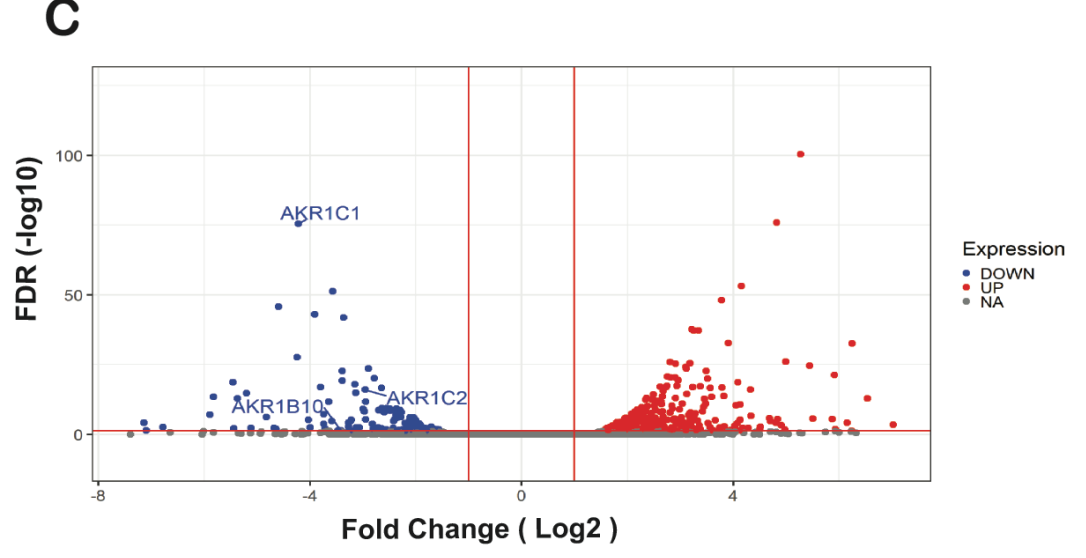

D

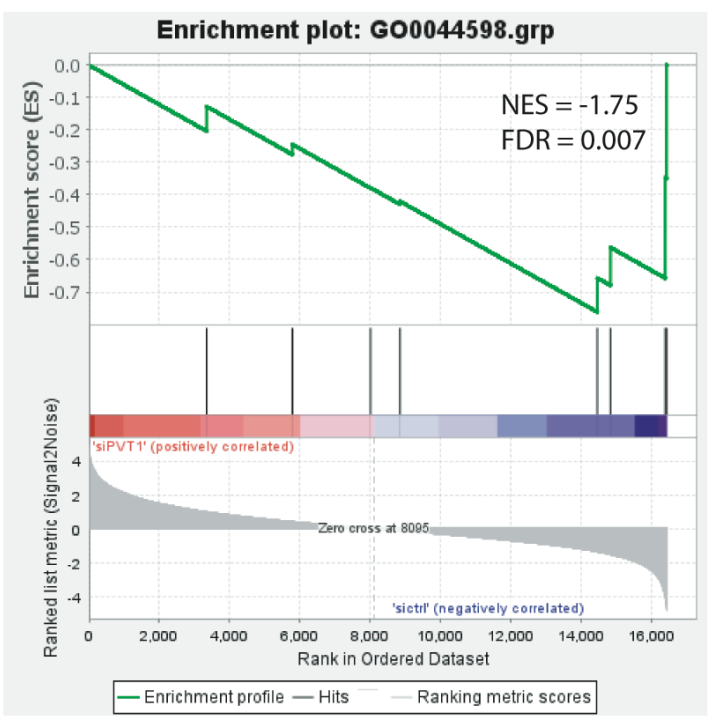

$\mathbf{E}$

\section{Doxorubicin metabolism genes}

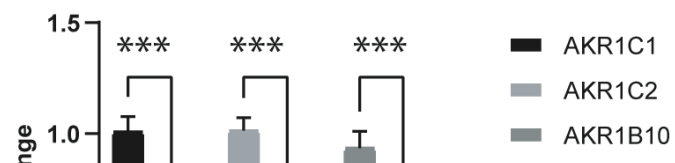

F

SK-OV3

ID8-IP2
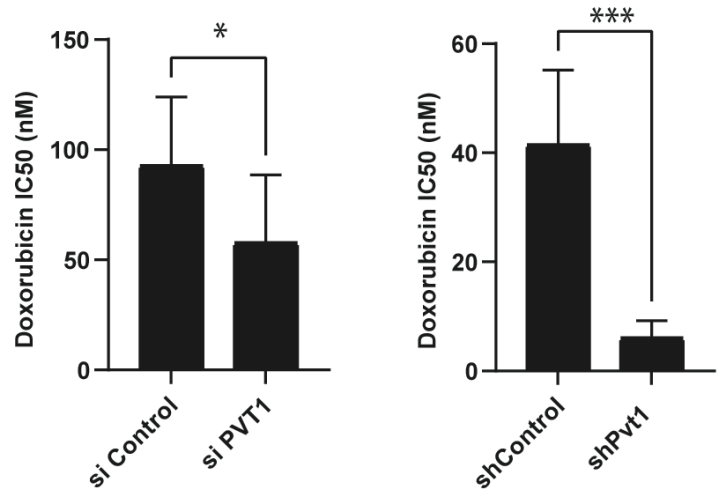


\section{Supplementary Figure 1}

Progression free survival_grade 2

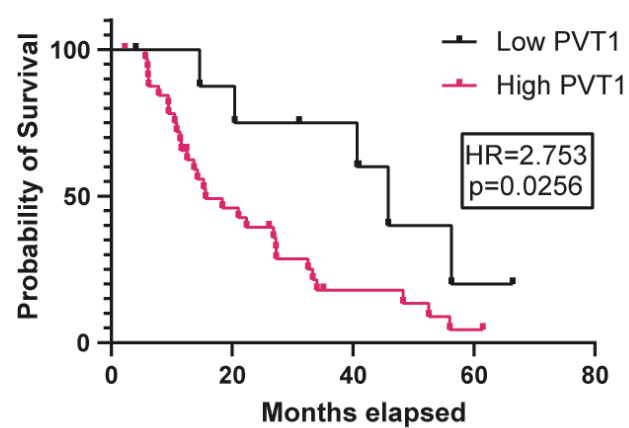

Progression free survival_grade 3

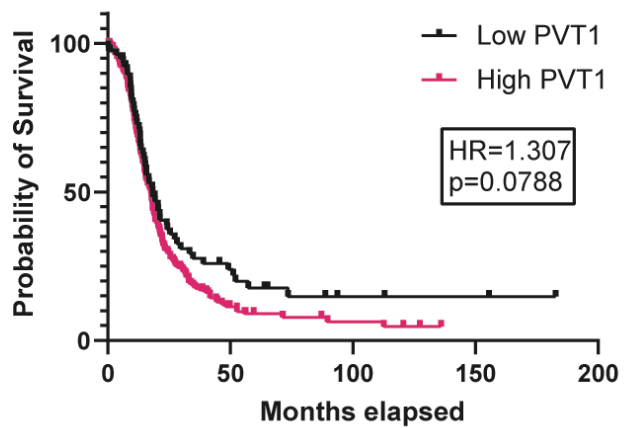

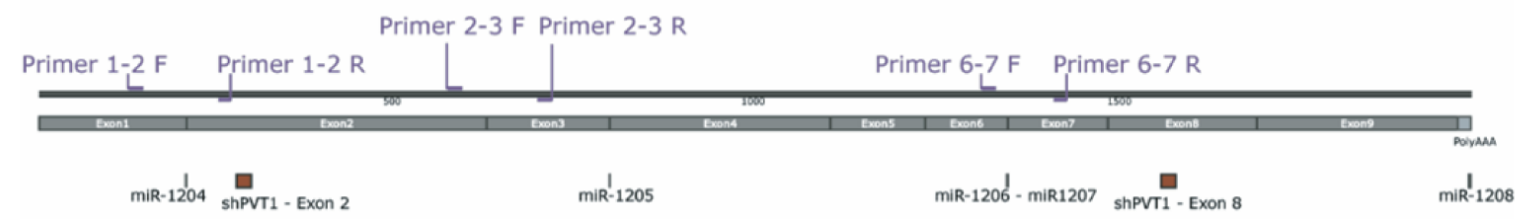

PVT1 mRNA (Human)

$1957 \mathrm{bp}$

Primer 2-3 F Primer 2-3 R

$\underset{\text { shpvt1 - } 1}{\square}$

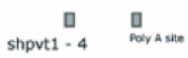

pvt1 mRNA (Mouse)

3076 bp

D

OV90 cell density

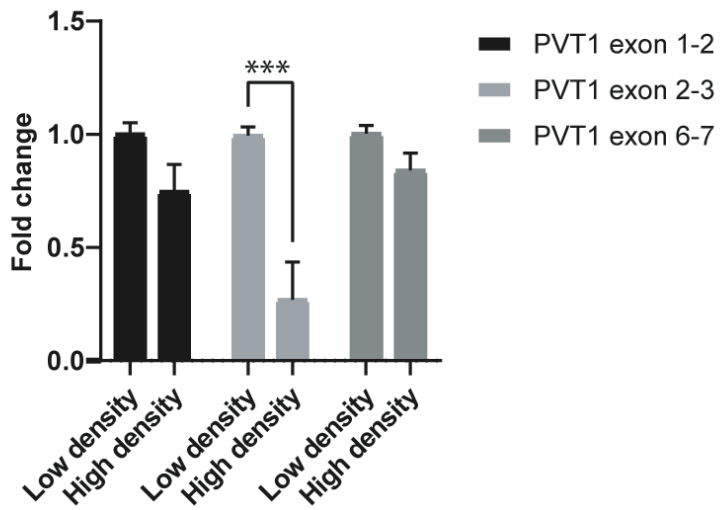

OVCAR3

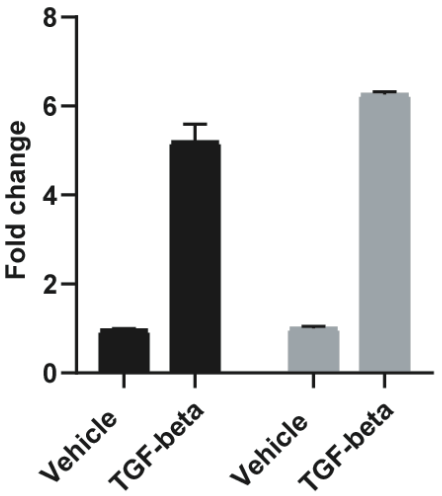

OVCAR420

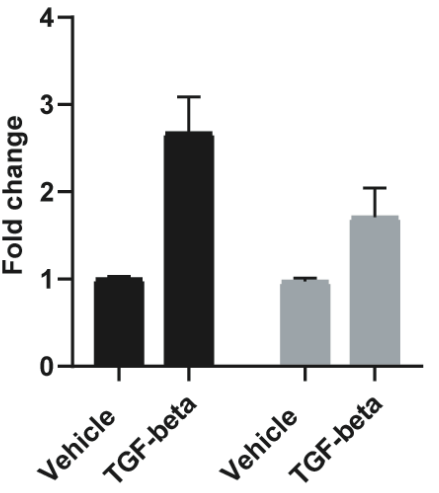

ZEB1

SNAIL1 
Supplementary Figure 2
A

\section{PVT1}

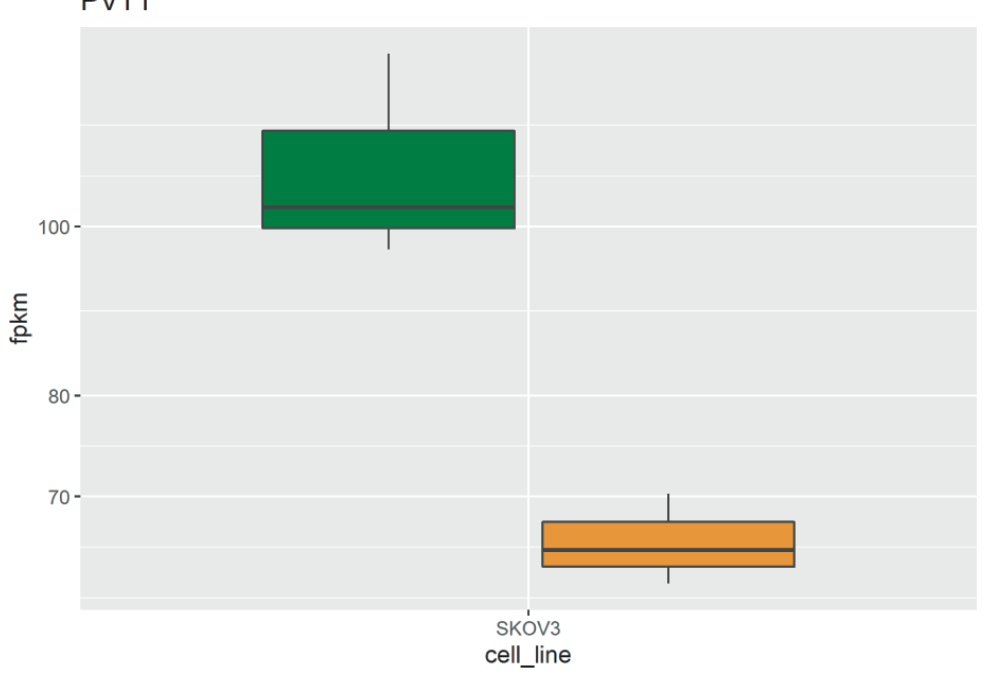

B

si_status

sicon

官 siPVT1

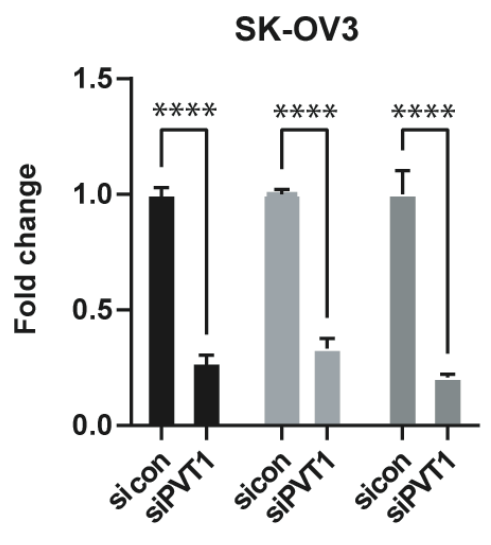

- PVT1 exon 1-2

- PVT1 exon 2-3

- PVT1 exon 6-7

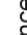
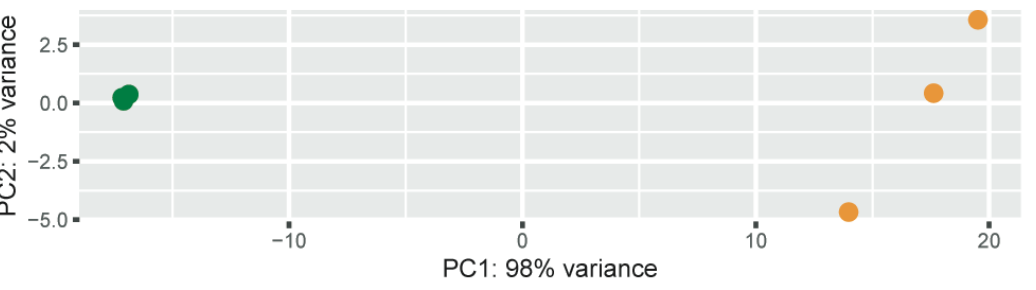

si_status

- sicon

- siPVT1 